\title{
Seafood Intake and Neurodevelopment: A Systematic Review
}

\author{
Claudia B. Avella-Garcia $•$ Jordi Julvez
}

Published online: 25 January 2014

(C) Springer International Publishing AG 2014

\begin{abstract}
Exposure to fish intake is of particular interest for neurodevelopment. Seafood contains nutrients that are essential for brain development and function. Seafood is also a potential source of well-established neurotoxic pollutants. We conducted a systematic search of the literature to review human studies on seafood intake and neurodevelopment. We identified 16 studies, most of them prospective cohort studies with prenatal and postnatal seafood intake exposure assessed through food frequency questionnaires. Most studies found positive associations with neurodevelopment outcomes, without particularly stronger associations for specific developmental areas (general, cognitive, and behavioral). Some studies observed an inverted U-shape association in relation with higher seafood-intake frequency. A few reports assessed type of seafood but no clear pattern was disentangled. In conclusion, seafood intake during pregnancy and postnatal periods seems to be beneficial to a wide range of neurodevelopment outcomes, with some potential risk at higher levels. Although
\end{abstract}

\author{
C. B. Avella-Garcia · J. Julvez $(\bowtie)$ \\ Centre for Research in Environmental Epidemiology \\ (CREAL)- PRBB, C. Doctor Aiguader 88, 08003 Barcelona, Spain \\ e-mail: jjulvez@creal.cat \\ C. B. Avella-Garcia $\cdot$ J. Julvez \\ IMIM( Hospital del Mar Medical Research Institute), Barcelona, \\ Spain \\ C. B. Avella-Garcia \\ Unitat Docent de Medicina Preventiva i Salut Pública H. \\ Mar-UPF-ASPB, Barcelona, Spain \\ C. B. Avella-Garcia • J. Julvez \\ Universitat Pompeu Fabra (UPF), Barcelona, Spain \\ J. Julvez \\ CIBER Epidemiologia y Salud Pública (CIBERESP), Barcelona, \\ Spain \\ C. B. Avella-Garcia \\ Universitat Autonoma de Barcelona (UAB), Barcelona, Spain
}

studies adjusted for a variety of sociodemographic factors, residual confounding is possible. Larger prospective studies are required to define which seafood species are more important for neurodevelopment while minimizing the potential neurotoxic effect of the related pollutants.

Keywords Fish intake · Pregnancy · Postnatal · Neurodevelopment $\cdot$ Seafood Chemicals $\cdot$ Review

\section{Introduction}

Essential nutrients are critical for brain development and function [1]. Humans have particularly long neurodevelopmental periods, and the human brain is in development until the early 20s. The early life period, however, is decisive because of the rapid and intense neurodevelopment processes that are fully activated during that time. The long-term consequences of disturbing neurodevelopment in early life by environmental and nutritional hazards can be enormous [2]. In the evaluation of the evidence on the protection of the developing human brain from environmental hazards, it is also important to consider potentially beneficial factors such as breastfeeding, physical activity and seafood intake [3].

An increasingly large number of epidemiologic studies have analyzed the association between seafood intake and neurodevelopment. Seafood contains high concentrations of long-chain n-3 polyunsaturated fatty acids (n-3 PUFA), which are essential nutrients for cell membrane formation, the development of neurons and their synaptic connexions [4]. Seafood contains other essential nutrients that are also required for normal brain function and development, such as selenium, vitamin $\mathrm{D}$, and iodine [5]. It is also, however, frequently contaminated by environmental pollutants including wellestablished neurotoxicants such as persistent organic pollutants (POPs) and mercury. These toxicants may be found in seafood because of their accumulation along the food chain 
from plankton to large predator fish (i.e., tuna and swordfish). The potential neurotoxicity of these compounds is of concern when formulating dietary recommendations on seafood intake for pregnant women and children [2].

Several reviews and systematic reviews have evaluated the role of POPs or mercury exposure with neurotoxicity [2, 6-9]. No previous reviews, however, have systematically evaluated epidemiologic studies on seafood intake early in life and neurodevelopment.

We conducted a systematic review of the evidence on the association of seafood intake during pregnancy and childhood with neurodevelopment. Our goal was to review the evidence in a systematic manner, to describe the limitations in exposure and outcome assessment of current research, and to discuss future research needs for improving the understanding of the complex connection between seafood and neurodevelopment. Additionally, we evaluated whether the published data support the recommendation of no more than $340 \mathrm{~g}$ per week of seafood during pregnancy issued by the US Federal Government Agencies [10]. We excluded from this review studies evaluating fish-oil supplements, measuring n-3 fatty acids, or evaluating specific seafood contaminants such as mercury or POPs if they did not report the overall association between seafood intake and neurodevelopment.

\section{Review Strategy}

We identified original articles studying the association of fish or seafood intake during the prenatal period, childhood and adolescence with neurodevelopment. Articles were searched in PubMed and PsycINFO databases using the following keywords: fish, seafood (as exposures), pregnancy, prenatal, uterine period, prenatal exposure delayed effects, maternal exposure, maternal-fetal exchange, infant, toddler, child, teenager, adolescent (as time periods), attention deficit, hyperactivity, neuro, neurological, mental health, cognitive abilities, neurodevelopment, neurobehavior, and behavior (as outcomes). Initially, a search of each of these terms was performed separately, and afterwards, a second search step combining the terms together was carried out by taking each term from the exposure group and crossing the search with each term from the time period group and each term from the outcomes group. The search period was limited to the last 20 years (from January 1993 until July 2013). Only human studies were included (Fig. 1). No language restrictions were implemented.

Identified abstracts were reviewed and articles were excluded using the following criteria: (1) not an original article, (2) non-human research, (3) not focused on seafood intake as one of the main exposures, (4) seafood exposure was measured in adulthood only, (5) no neurodevelopmental endpoint, (6) duplicated between databases. Among 20 articles identified and fully reviewed, 3 were further excluded because they did not meet the criteria for inclusion [11-13]. We also excluded a randomized control trial of a fish flour bread spread because of the different nature of the exposure compared to observational studies of seafood intake $[14 \bullet \bullet$. The two authors reviewed the articles independently, and differences in opinion regarding inclusion or exclusion of the articles were settled by consensus between them. A manual review was performed by scrutinizing the reference list of each one of the retrieved studies. No additional articles were identified through manual search.

For each study included we collected the following data: authors, journal, year of publication, study design, study population, study period, sample size, outcome assessment, age at outcome assessment, seafood assessment, and measure of association and confidence intervals in the most adjusted model and covariates controlled for in the analyses (Tables 1 and 2). Most studies reported estimated measures of relative risk (odds ratios, relative risk). For studies that reported associations with both continuous and dichotomous measures of the study outcome, we selected the results for the dichotomous measures only [15]. Some studies reported findings only for continuous measures of the outcome, and we report those results (mainly $\beta$ coefficients). For the subset of studies that adjusted the association between seafood intake and neurodevelopment outcomes for mercury and PCBs, we report the findings both before and after adjustment for those established neurotoxicants in a separate table (Table 3).

\section{Results}

\section{Study Characteristics}

A total of 16 articles were found (Tables 1 and 2). Eight studies evaluated seafood exposure prenatally, five studies evaluated seafood exposure postnatally, and three studies evaluated seafood exposure both pre- and postnatally. After birth, seafood intake was evaluated during childhood [1, 14••, 15-17, 18•] and adolescence [19-21]. Most of the studies were prospective cohorts $[1,10,15-17,18 \bullet, 20-26,27 \bullet]$, probably the best-suited methodology to evaluate whether maternal intake of fish can influence the development of the offspring. Other study designs were cross-sectional [19] and case control [28] .

The studies were from different geographical regions, although predominantly from developed countries. Most studies have been carried out in Europe [1, 10, 15, 16, 18 • 19-21, 23, $25,28]$, followed by others in the US [22, 27•], Japan [26], and New Zealand [17]. Almost all of them were conducted in general population groups [1, 10, 15-17, 18•, 22-26, 27•]. Most studies used a Food Frequency Questionnaire (FFQ) [1, $10,15-17,18 \cdot, 22-26,27 \bullet, 28]$ to assess dietary seafood 
Fig. 1 Flow chart of study selection

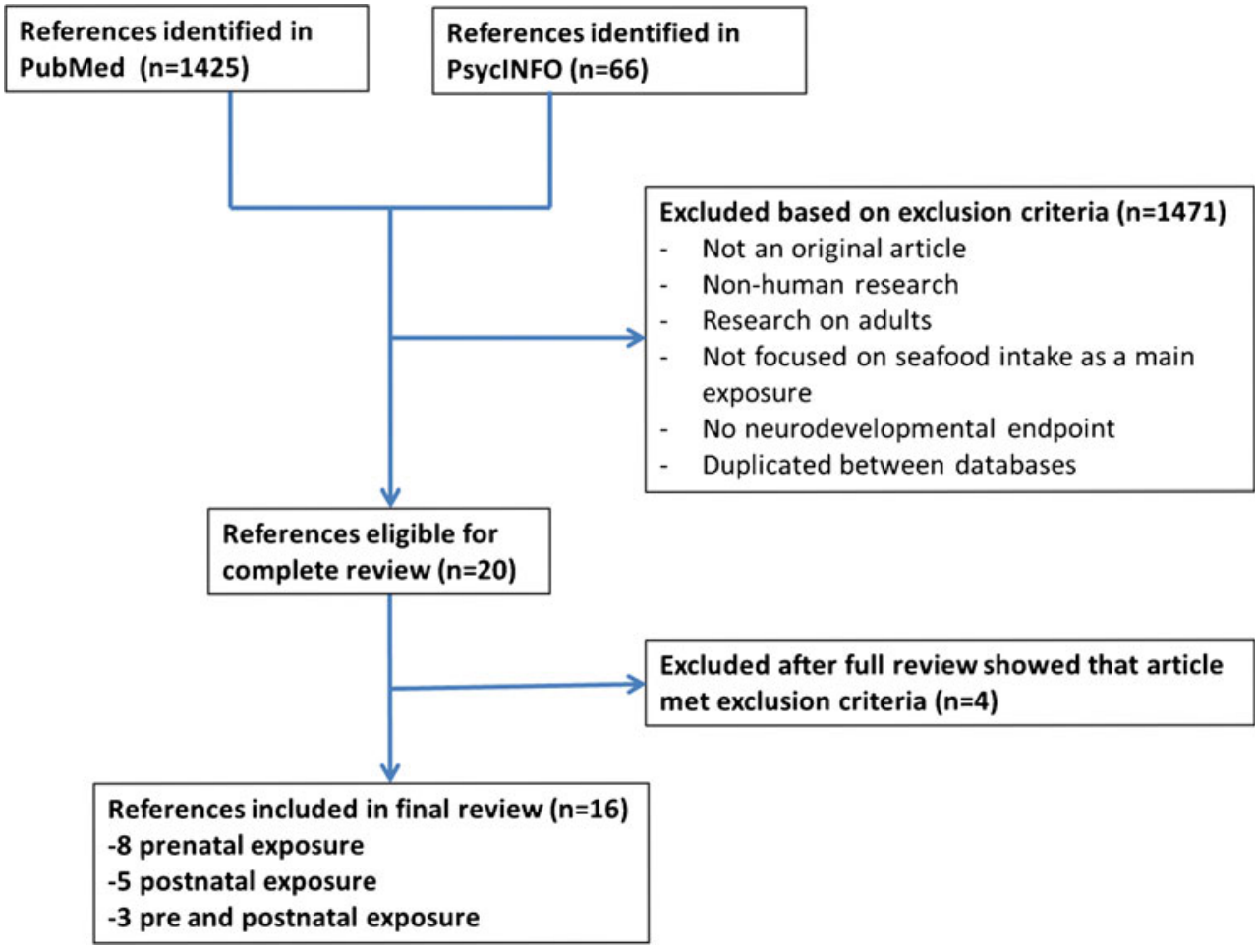

intake, especially for studies examining prenatal exposure. Other dietary questionnaires were also used in postnatal exposure studies [15, 16, 19-21], ranging from simple questions to dietary diaries (Table 2).

The neurodevelopmental outcomes and the tools used for outcome assessment varied widely across studies. Outcomes can be classified as pertaining to cognitive, behavioral, or general developmental areas. Cognitive outcomes were ascertained by using VRM (Visual Recognition Memory) [22], WISC (Wechsler Intelligence Scale for Children) [10, 17, 25, 27•], PPVT (Peabody Picture Vocabulary Test), WRAVMA (Wide Range of Visual Motor Ability) [24], MSCA (McCarthy Scales of Children's Abilities) [1, 18•], AVT (Amsterdam Vocabulary Test) [19], Swedish military conscription intelligence test [21], Stanford-Binet intelligence scales [17], CPT (Continuous Performance Test) [27•], or school grades [19, 20]. Behavioral outcomes were measured by the SDQ (Strengths and Difficulties Questionnaire) [10, 25], CRS-T (Conner's Rating Scale-Teacher's version) [27•], and the YSR (Youth Self-Report) [19]. General development tests included: the stereoacuity test [16], MCDI (MacArthur Communicative Development Inventory) and DDST (Denver Developmental Screening Test) [15], ALSPAC scale (Avon Longitudinal Study of Parents and Children) [10], NBAS (Neonatal Behavioral Assessment Scale) [26], neurological exam [28], and maternal interview.

Common covariates were child age $[15,19,22-24,27 \bullet$, 28], sex [1, 10, 15, 16, 19, 22-24, 26, 27•], and breastfeeding $[1,10,15,16,18 \bullet, 22-25]$, as well as maternal age $[10,15,16$,
$22,24,25,27 \bullet, 28]$, sociodemographic characteristics [1, 10, 15-17, 18•, 19, 21-26, 27•], smoking [10, 15-17, 18•, 24-26, $27 \bullet]$, and alcohol use [10, 15, 24-26, 27•]. Of special interest, seven articles also reported having adjusted in some way for the possible effects of contaminants such as mercury $[15,18$, $22,24,26,27 \cdot]$ or persistent organic pollutants $[1,26,27 \bullet]$ (Table 3 ). In some but not all studies, adjusting for mercury and/or POPs further strengthened the positive association between seafood intake and neurodevelopmental outcomes.

\section{Studies of Prenatal Seafood Intake}

In the cohorts that studied prenatal maternal seafood intake, the ages of children at testing ranged from days to 10 years (Table 1). Prenatal exposure to seafood as part of the maternal diet was found to have a positive relation with general developmental outcomes including improved scores on the MCDI and DDST scales at 18 months [15], higher scores on the ALSPAC scale from 6 to 42 months of age [10], having achieved foveal stereoacuity (an indicator of visual cortex maturity at 3.5 years of age; the association was only significant for oily fish) [16] and acquirement of developmental milestones at 6 and 18 months [23]. A Japanese study found no significant differences in newborns [26].

Seafood intake was also positively associated with cognitive benefits in several studies including higher verbal IQ at 8 years [10], higher full-scale IQ in children at 9 years [25], better results for VRM at 5.5-8.4 months of age [22] (which was strengthened after adjustment for maternal hair mercury 


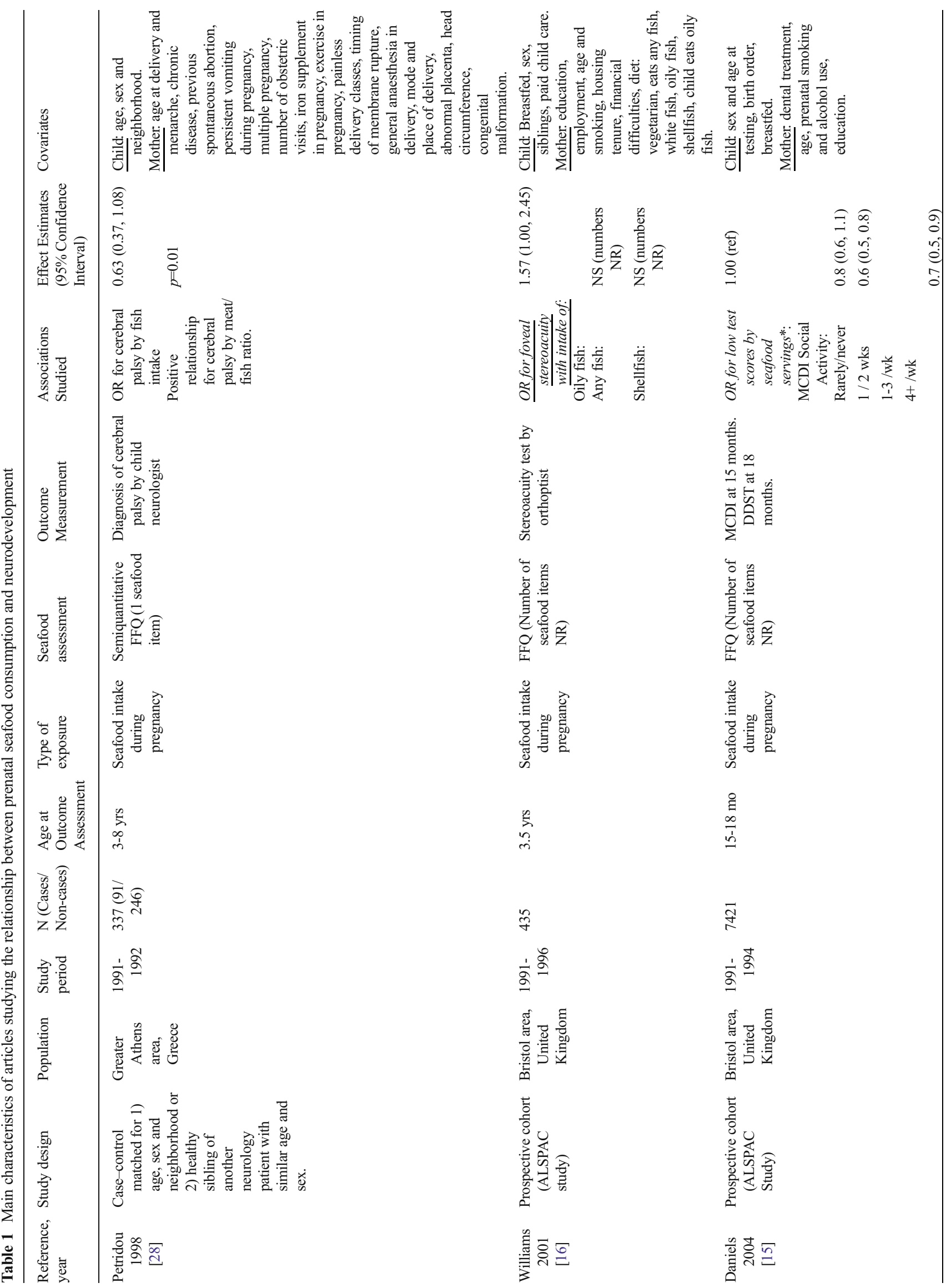




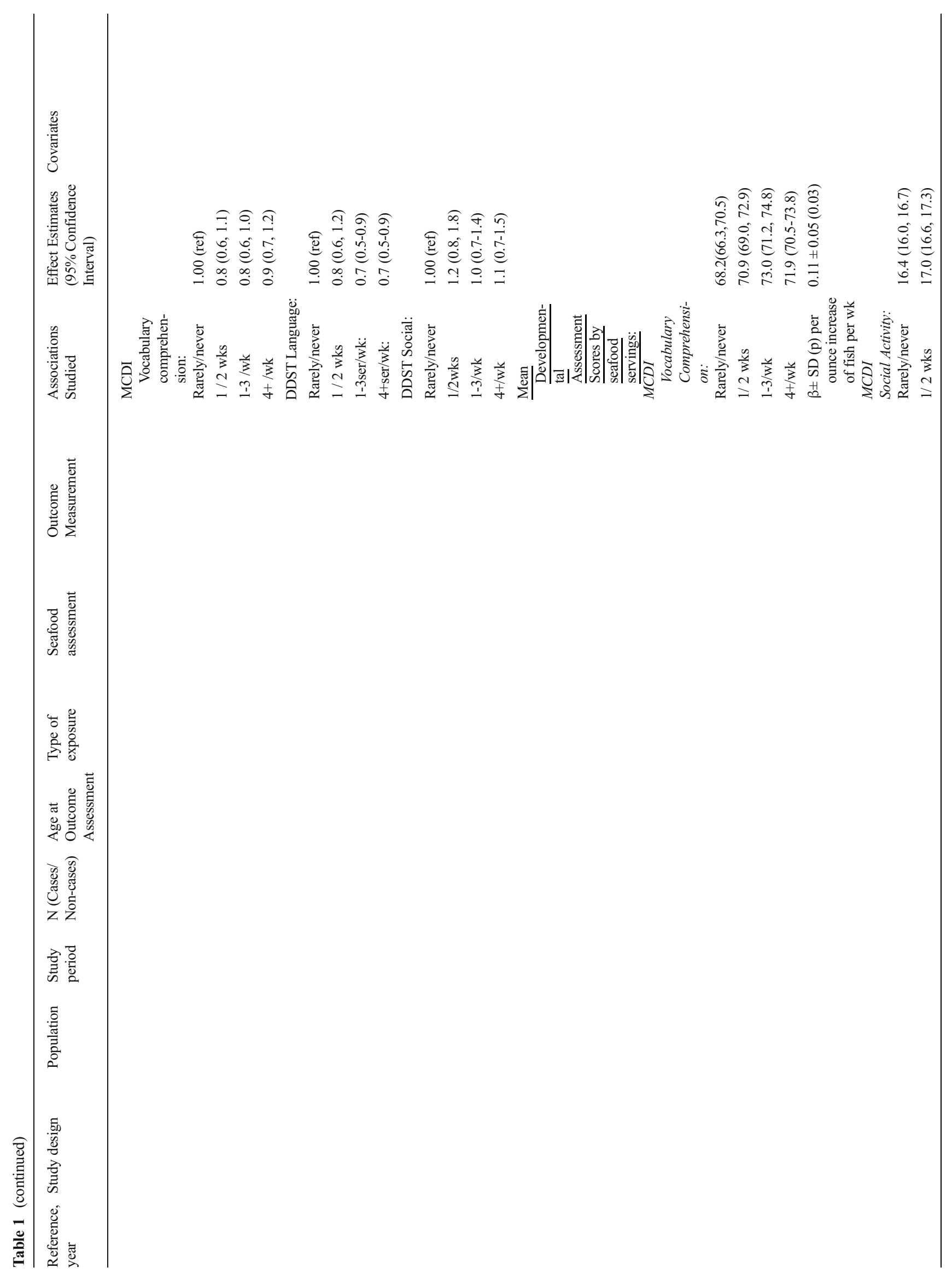




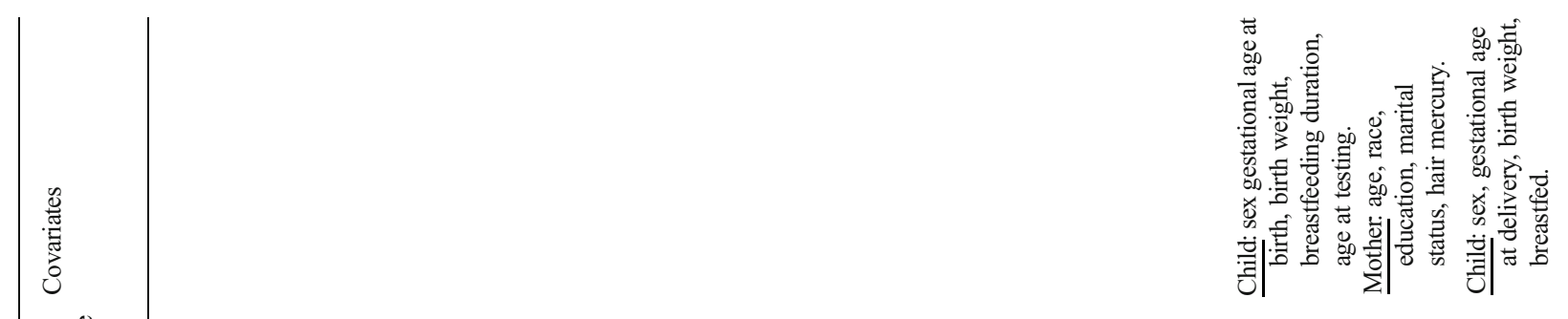

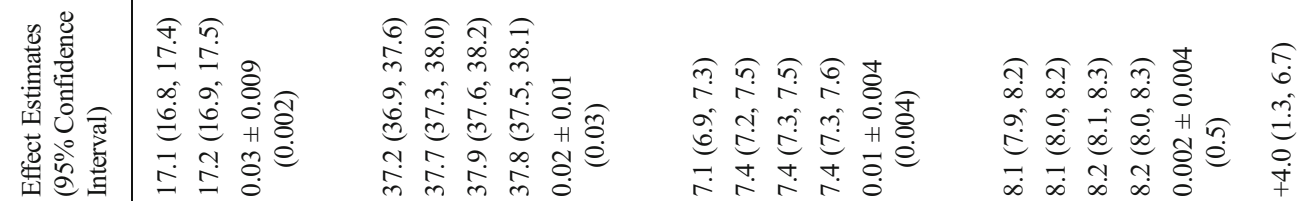

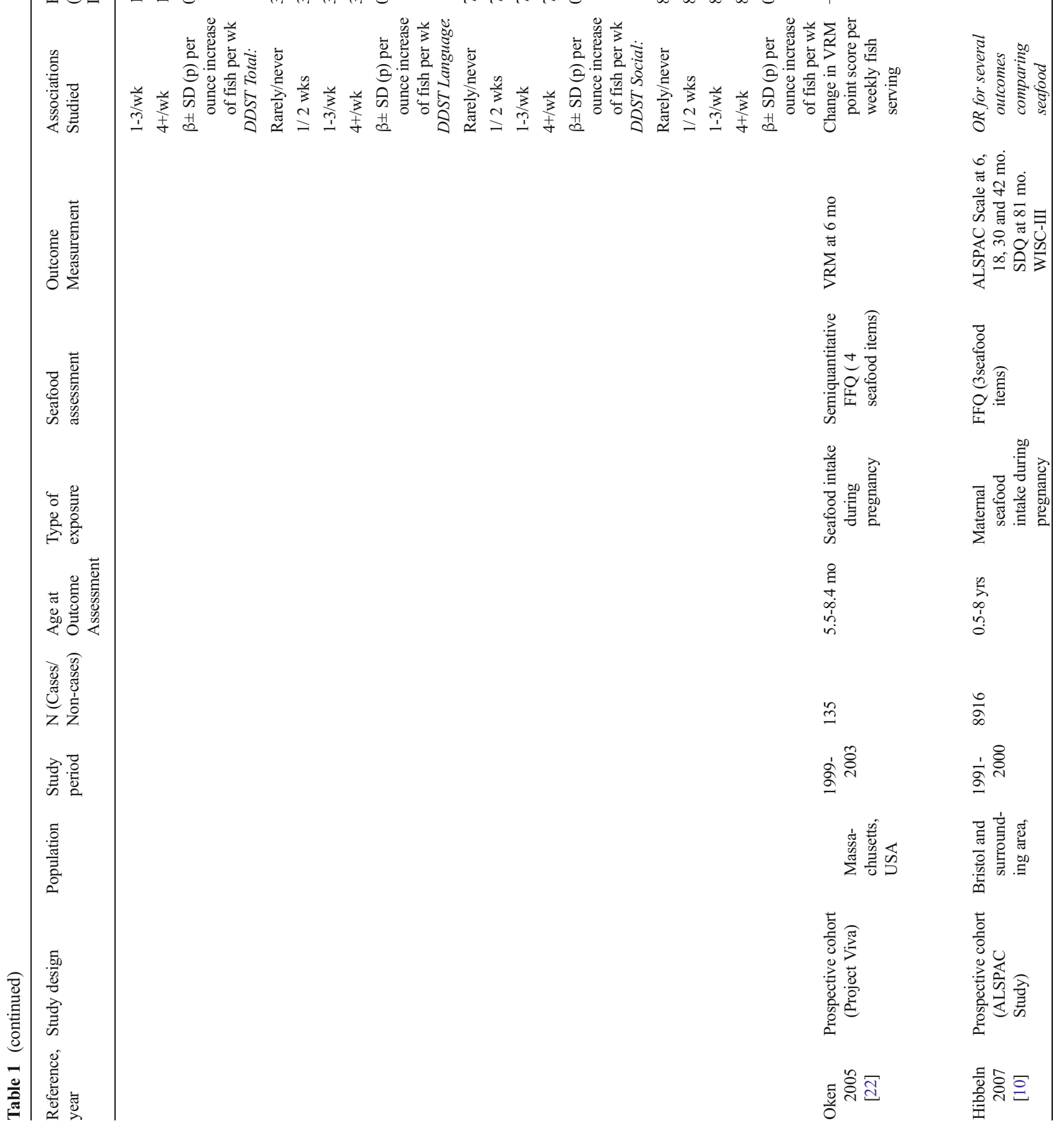




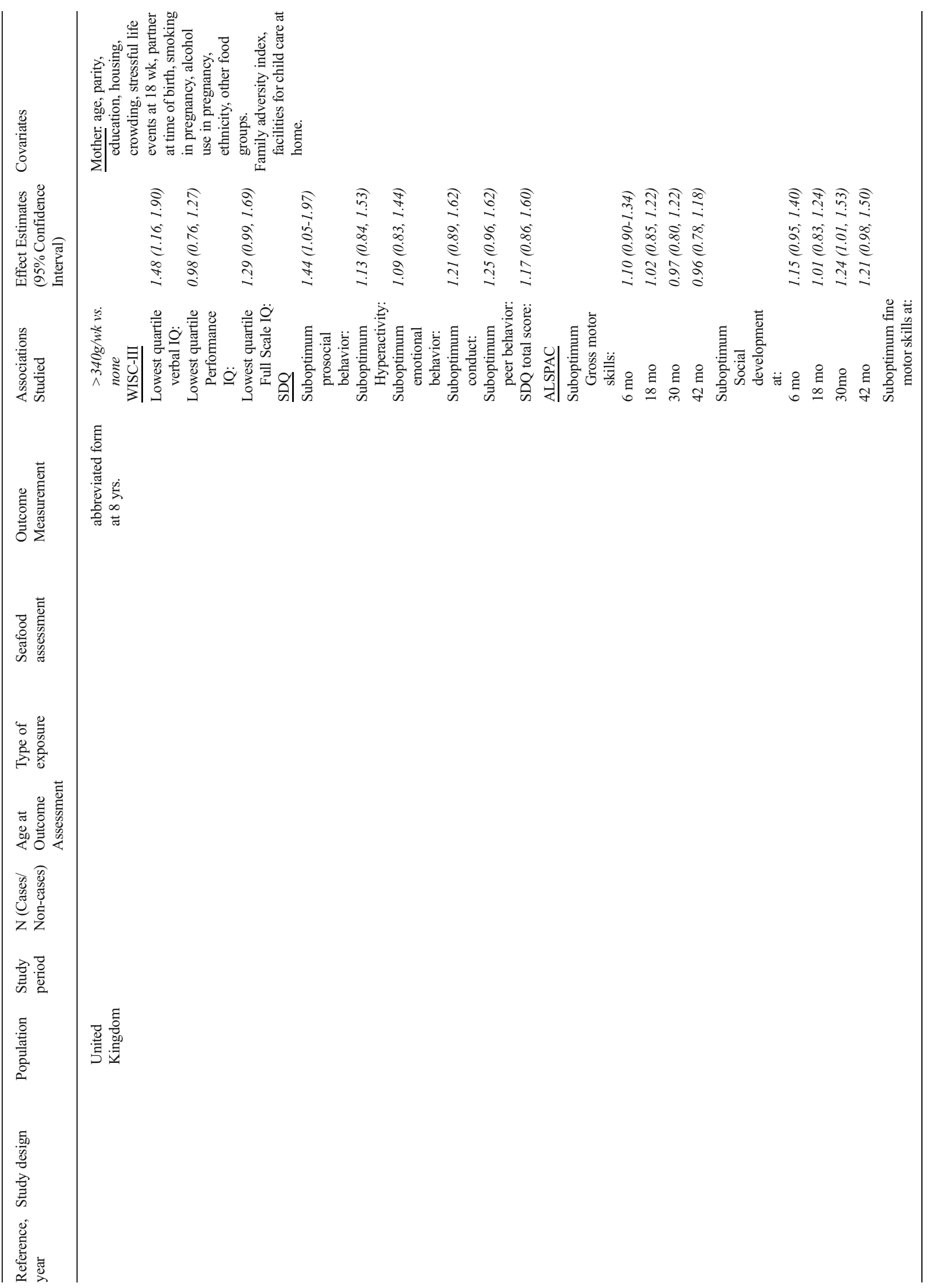




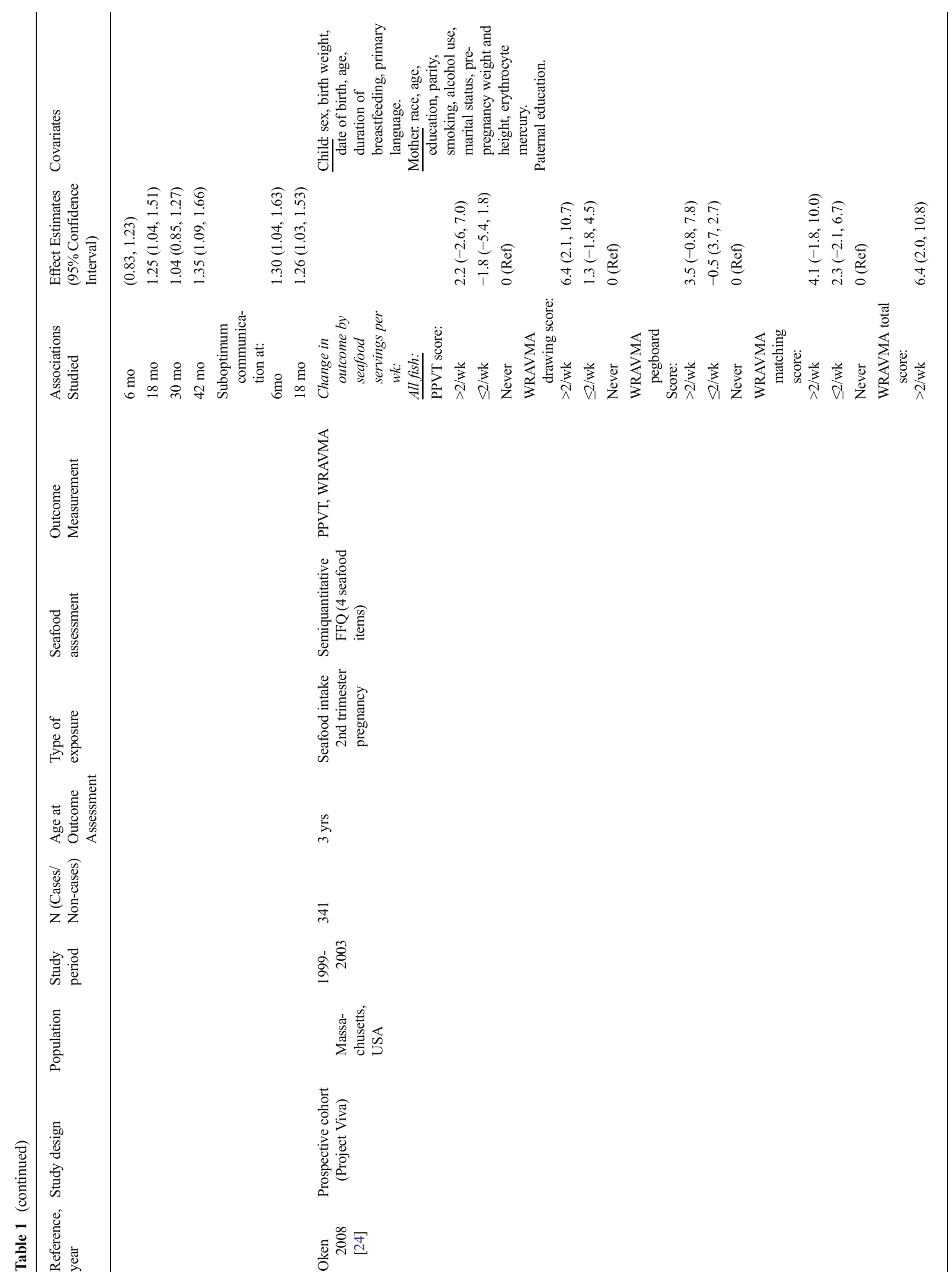




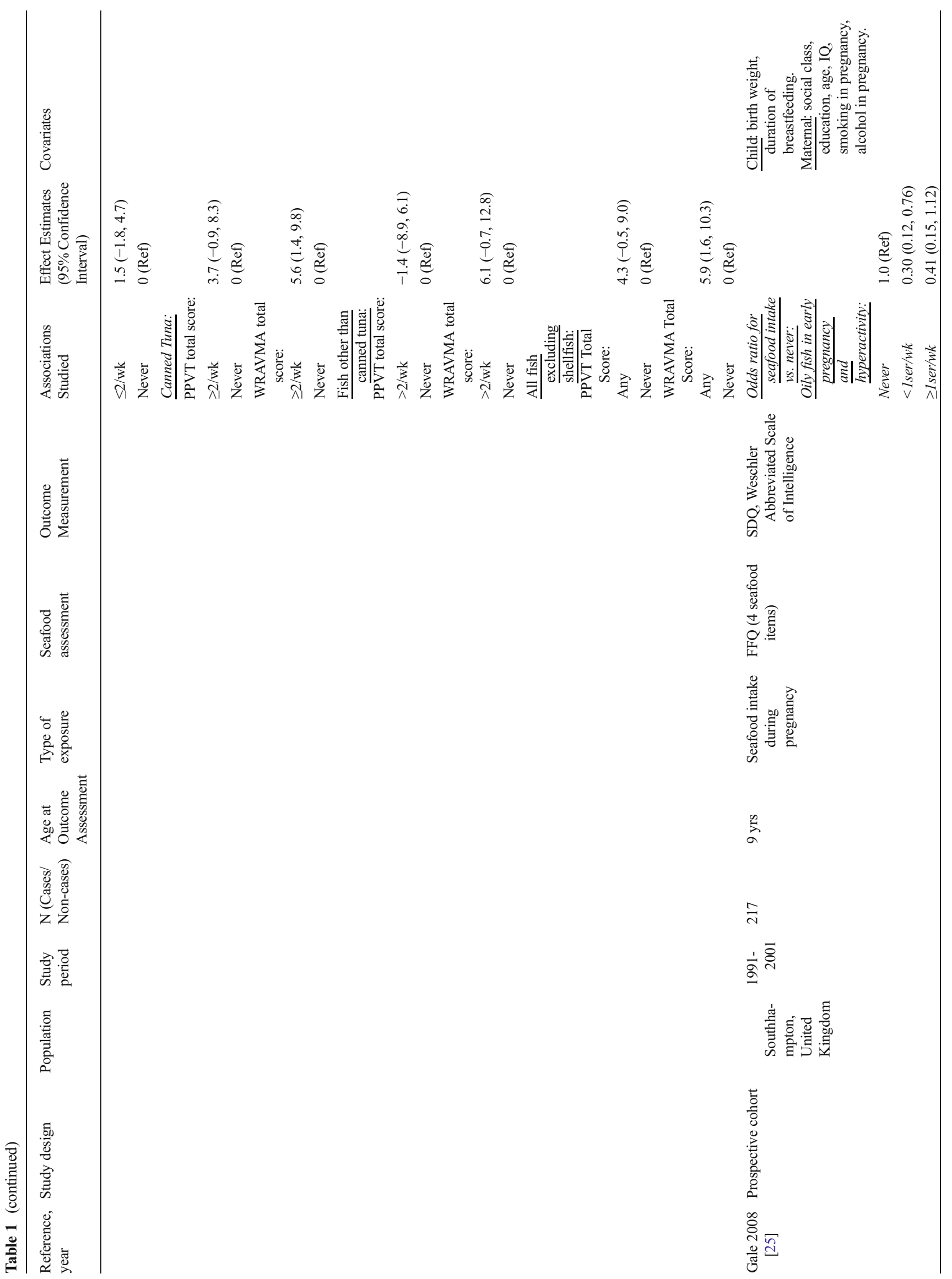




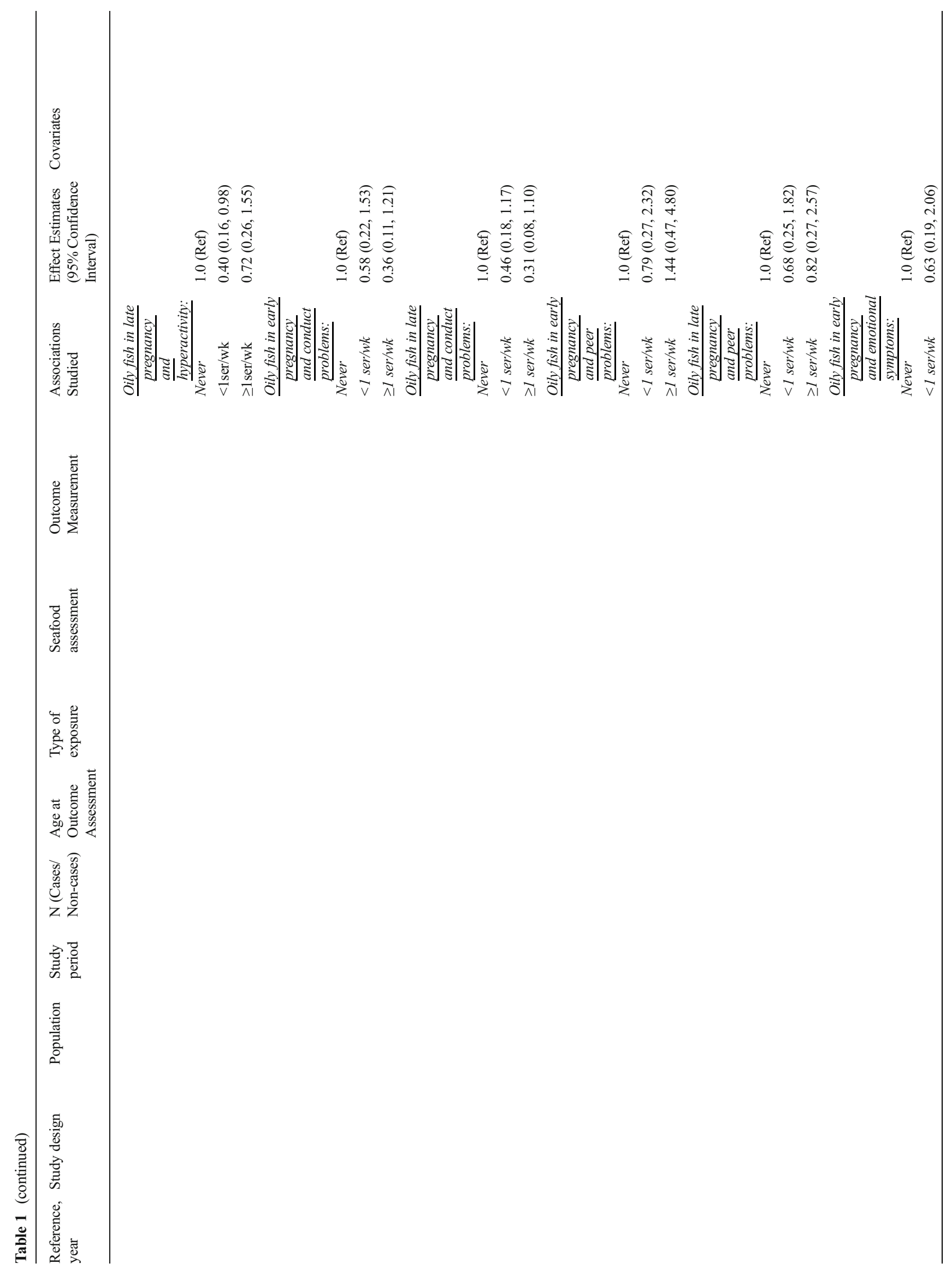




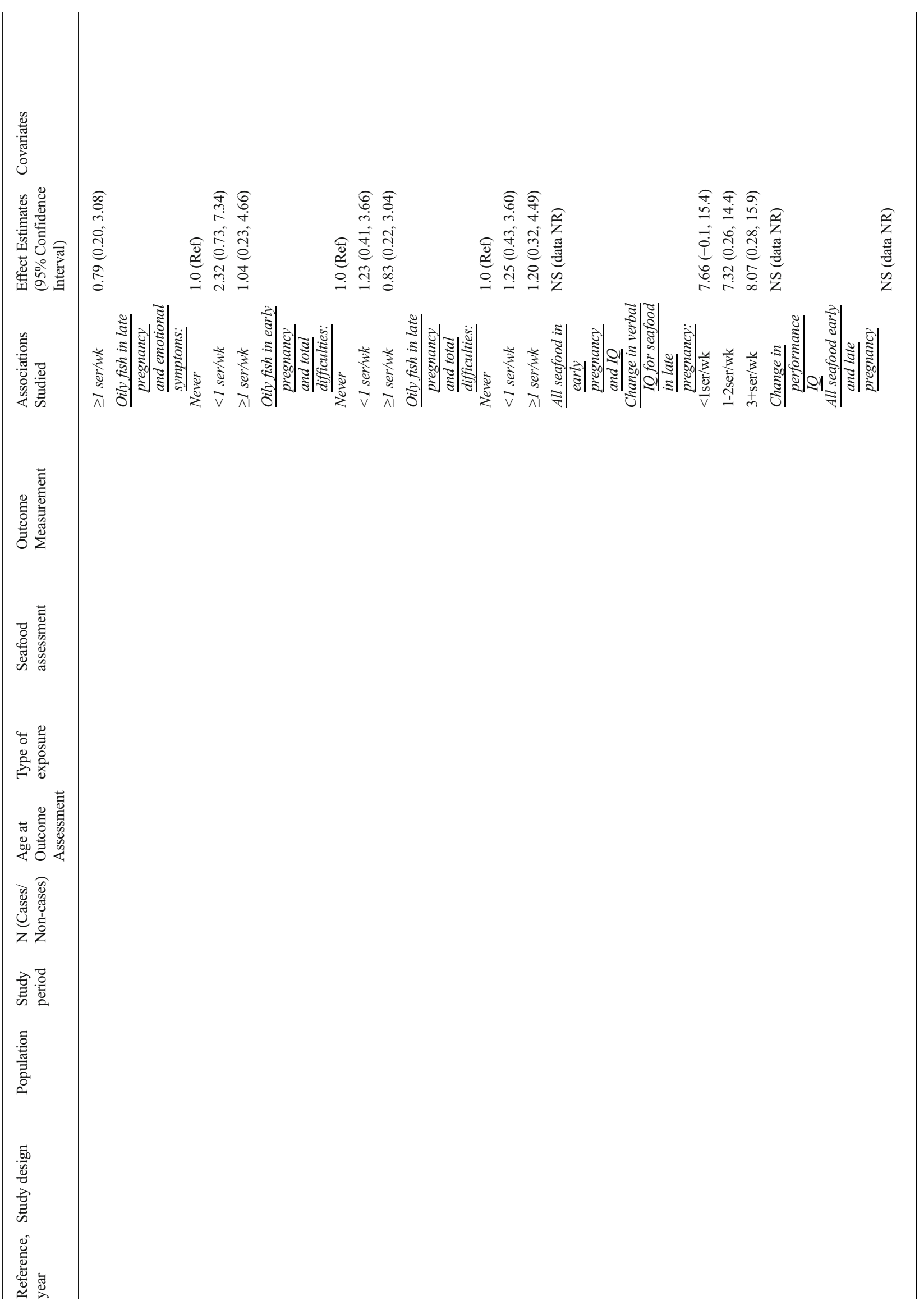




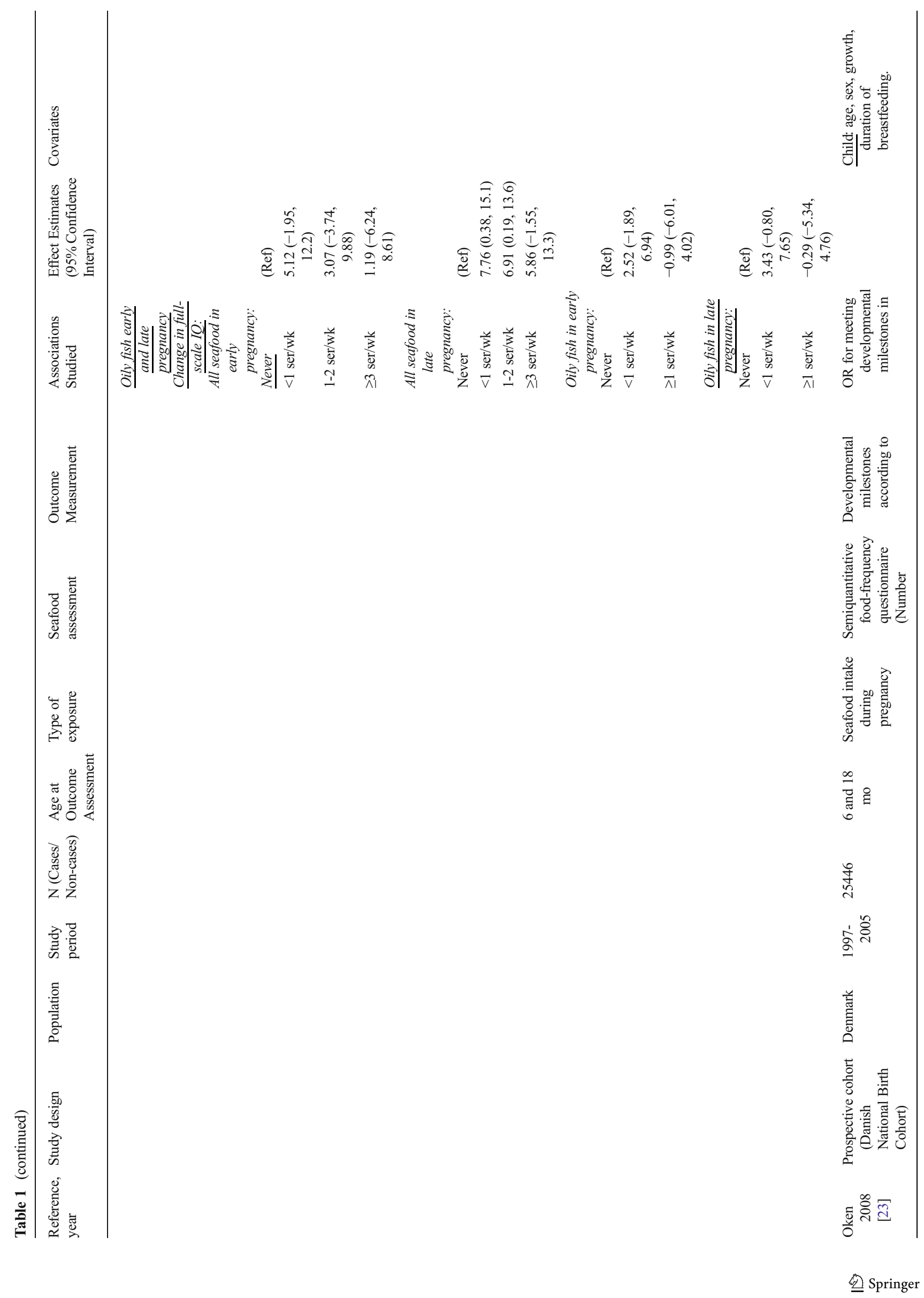




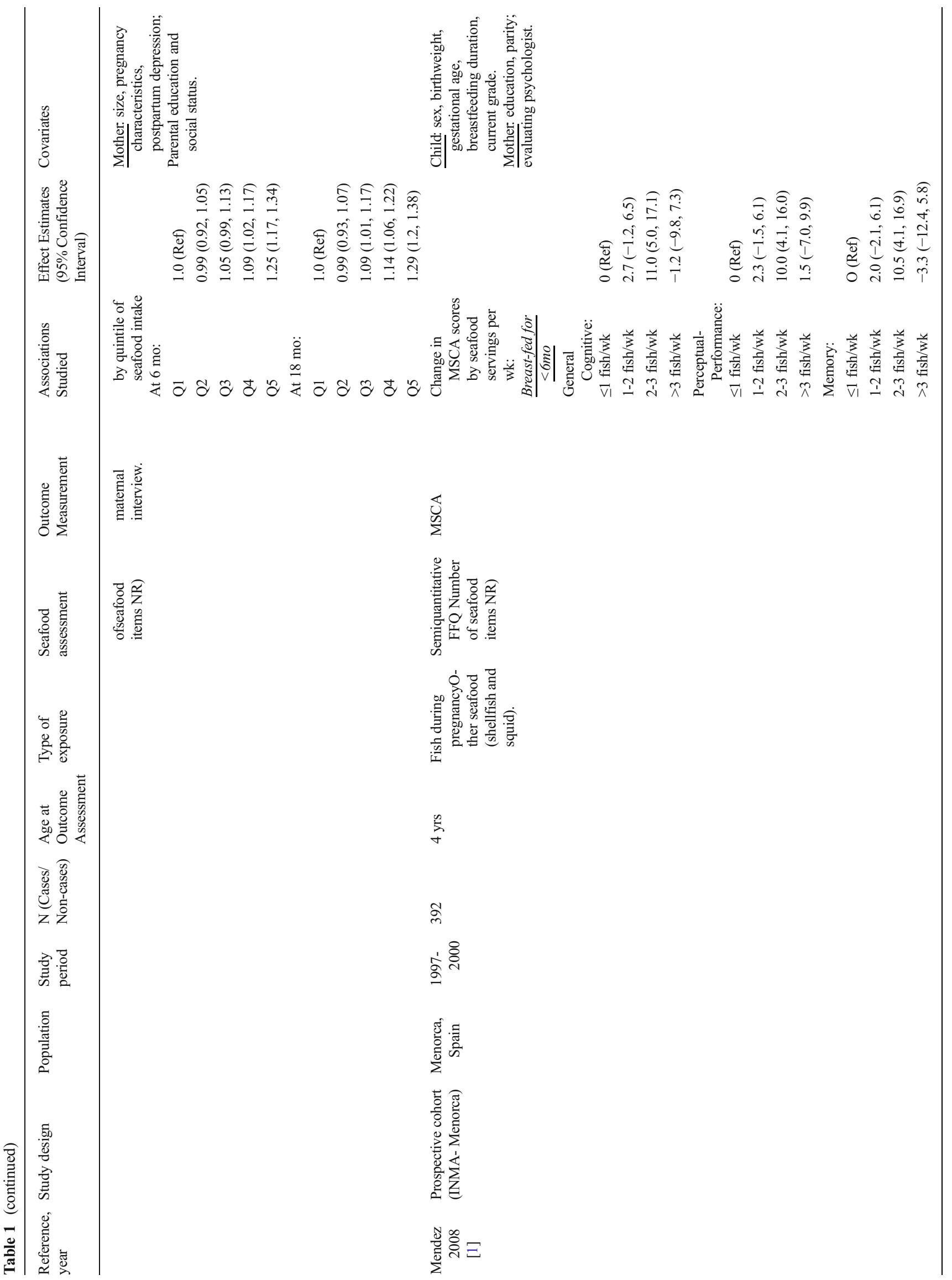




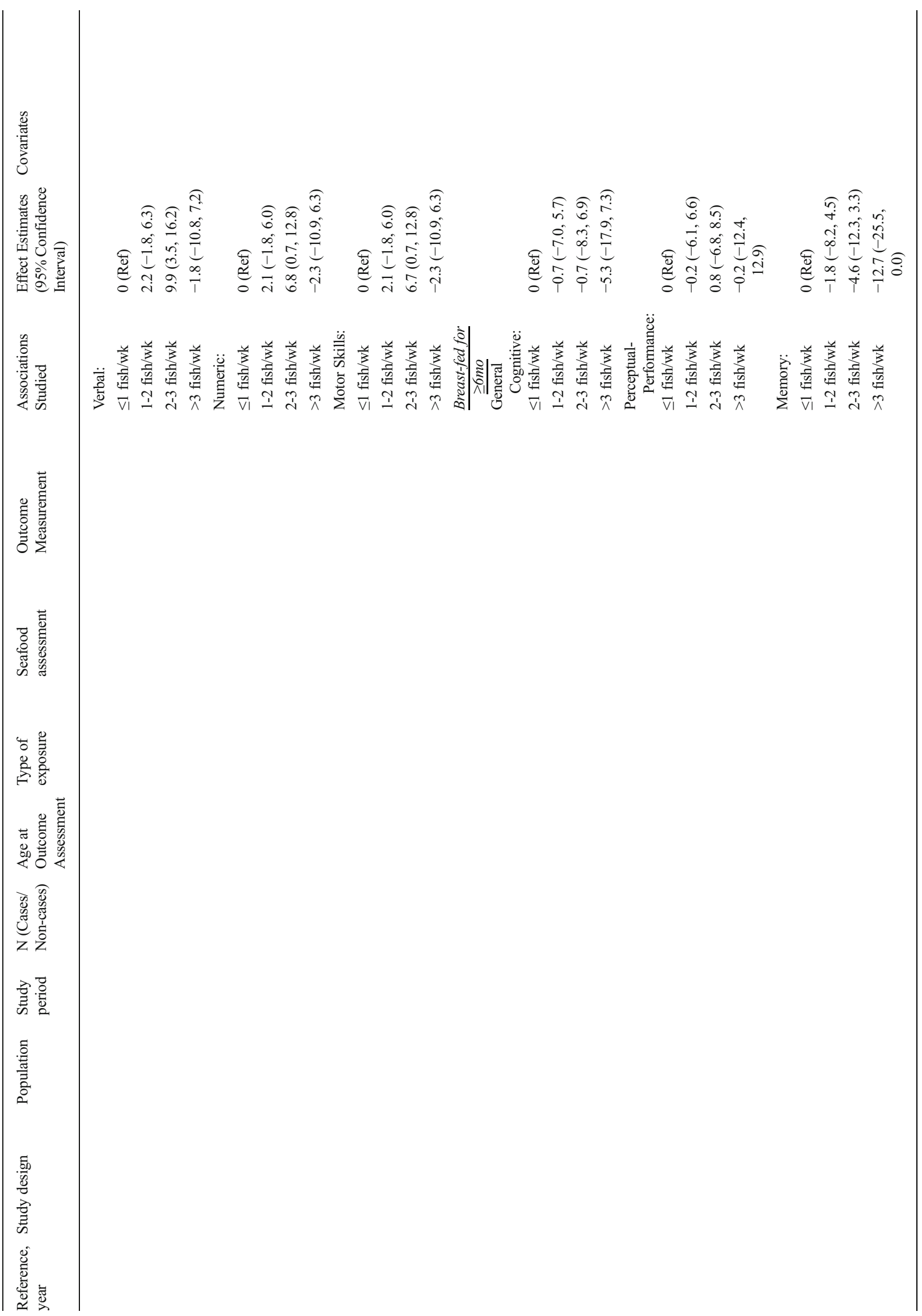




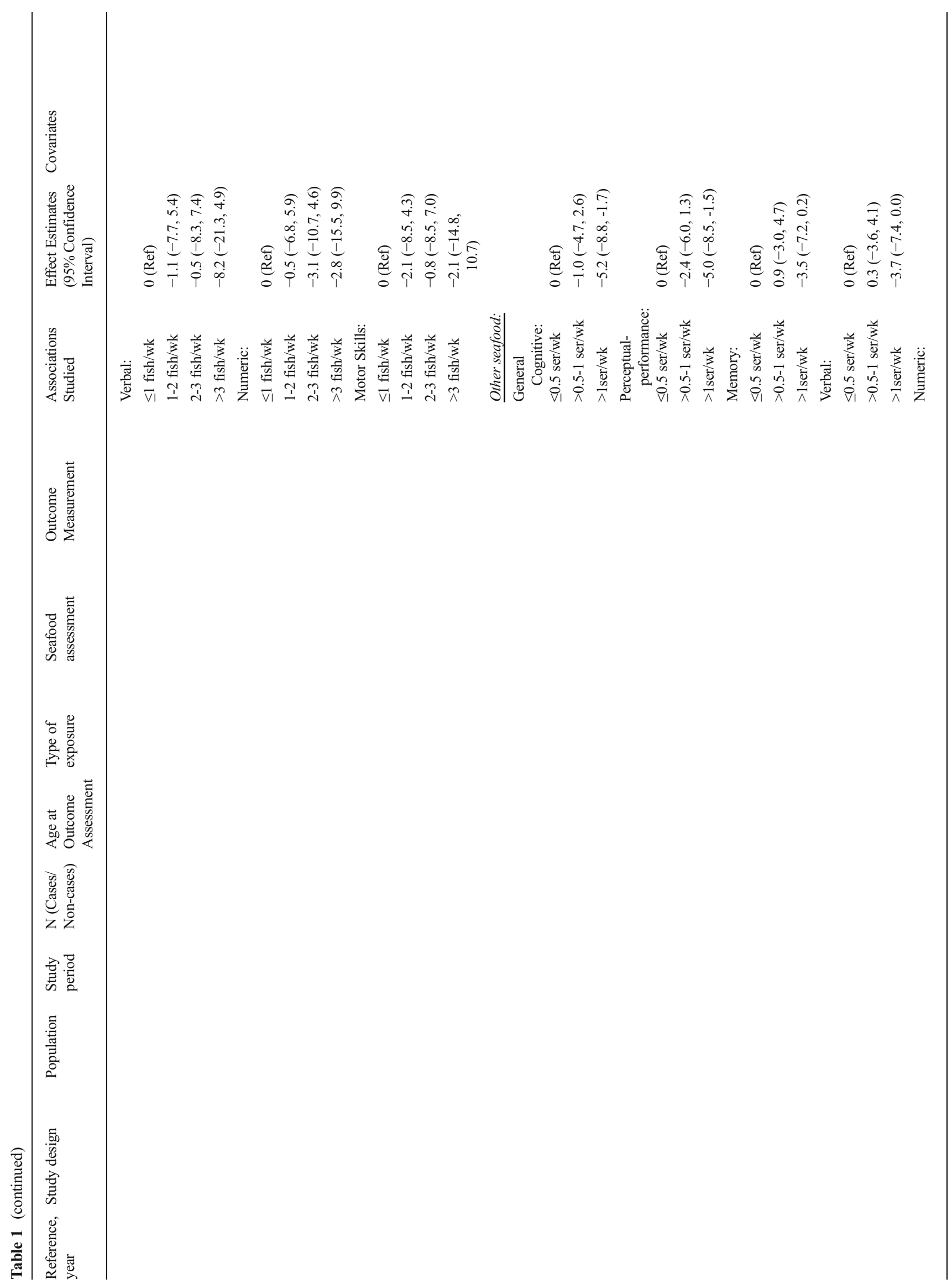




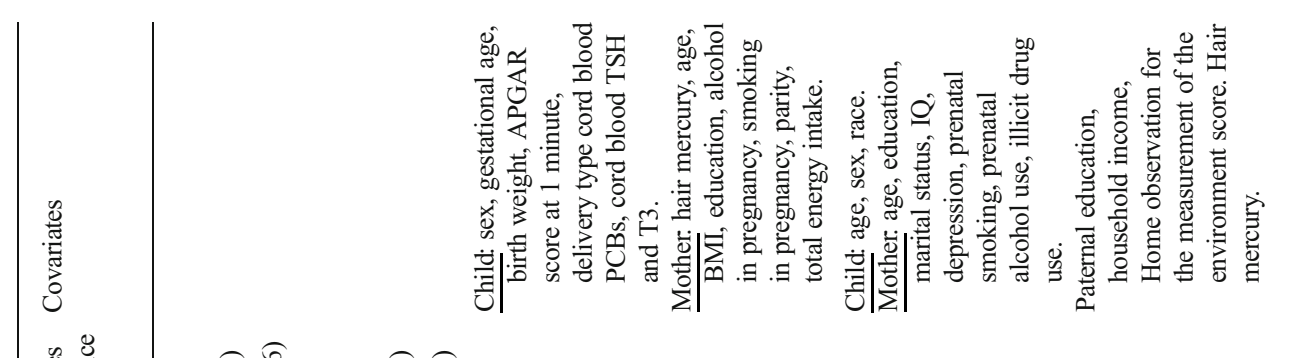

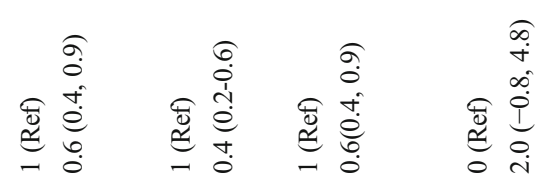

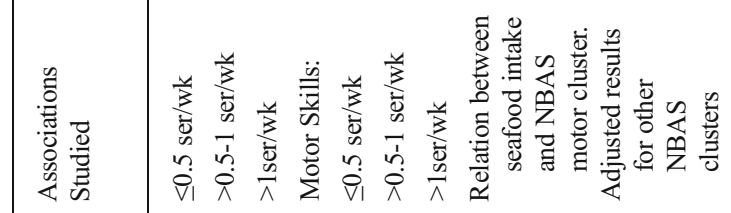

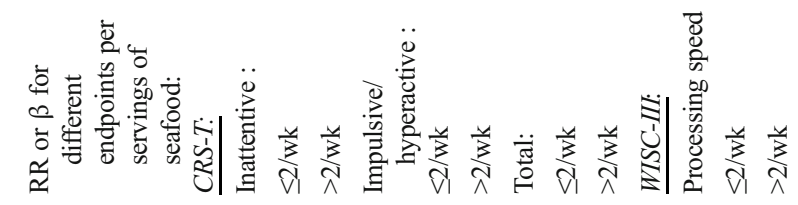

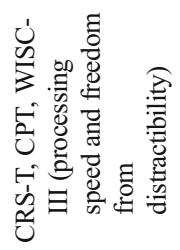

है
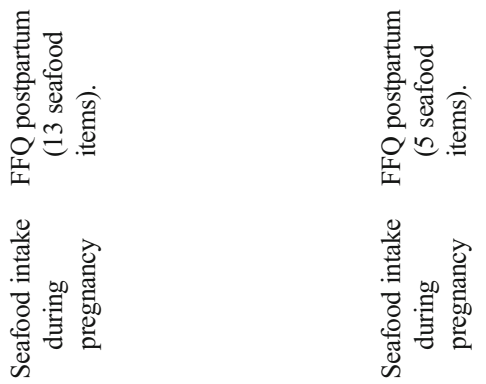

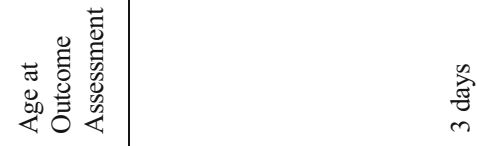

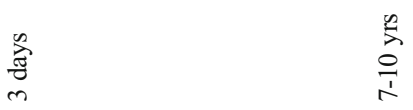

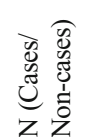

$\stackrel{\leftrightarrow}{\stackrel{\gamma}{*}}$

$\frac{5}{\frac{2}{1}}$

宫

$\frac{n}{n}$

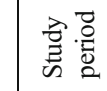

高

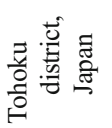

๙ूँ

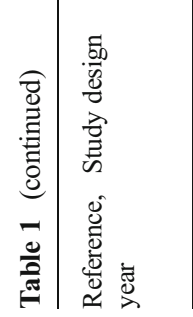

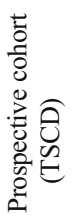

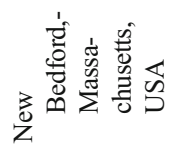

放

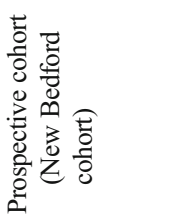

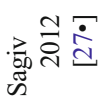




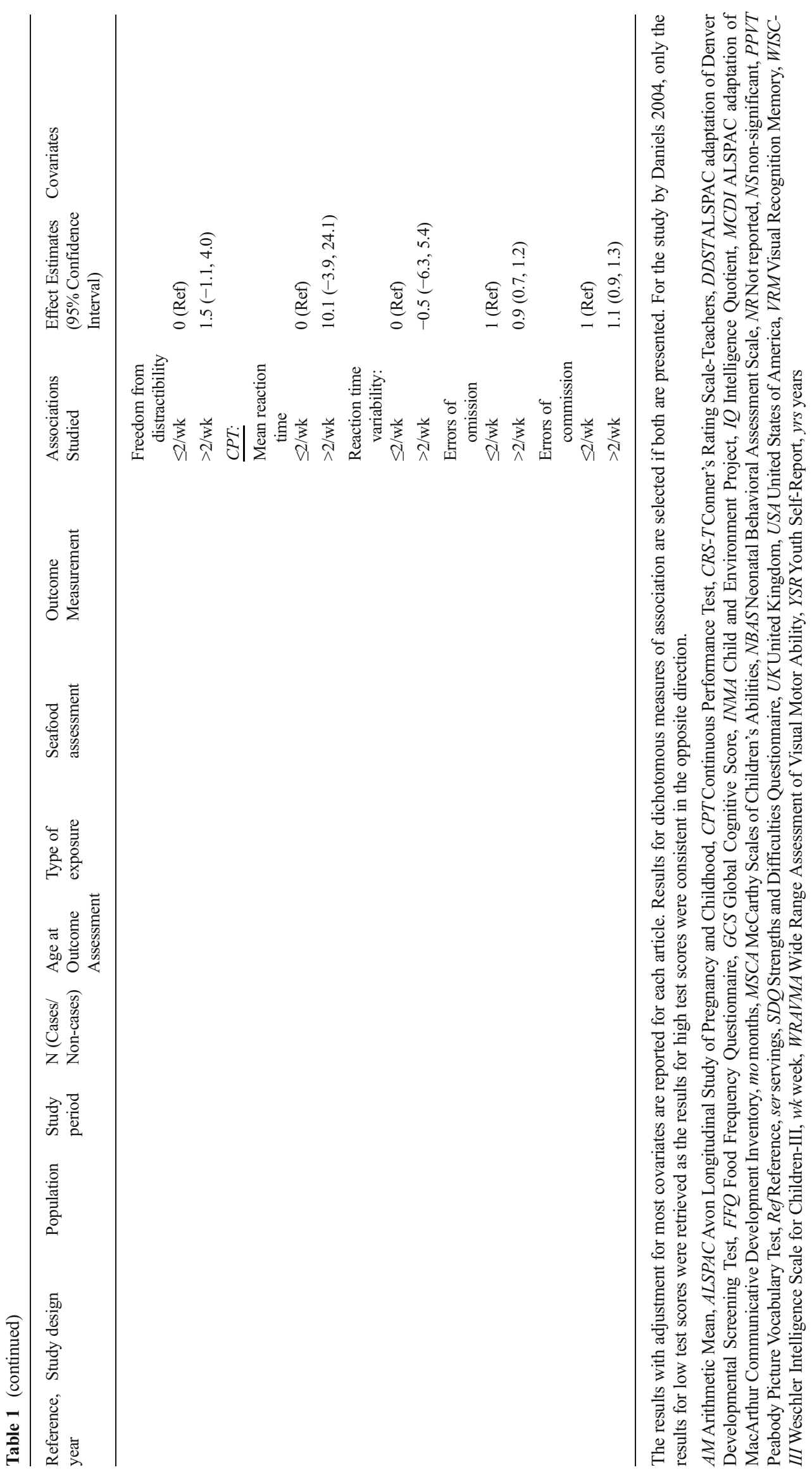




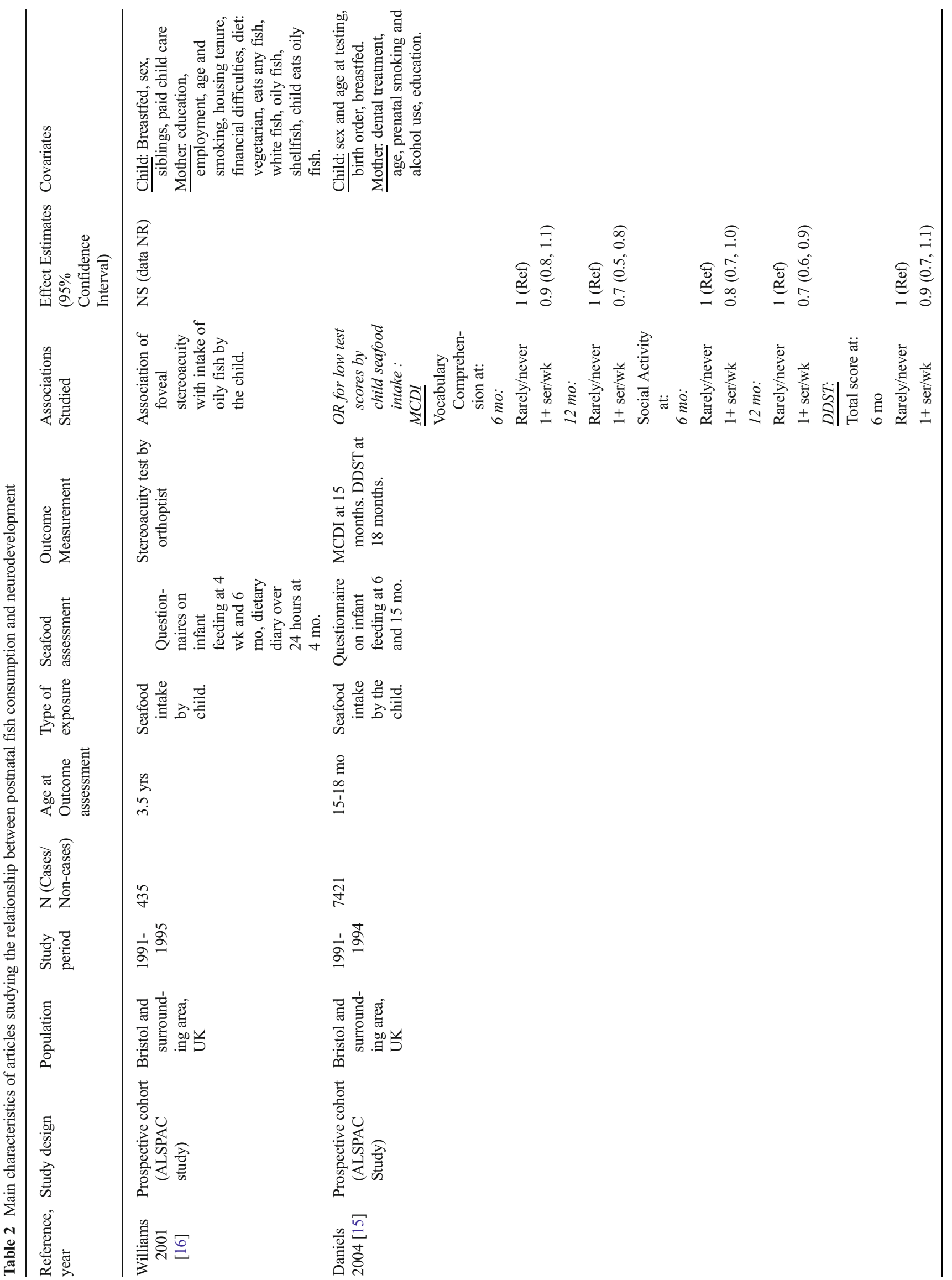




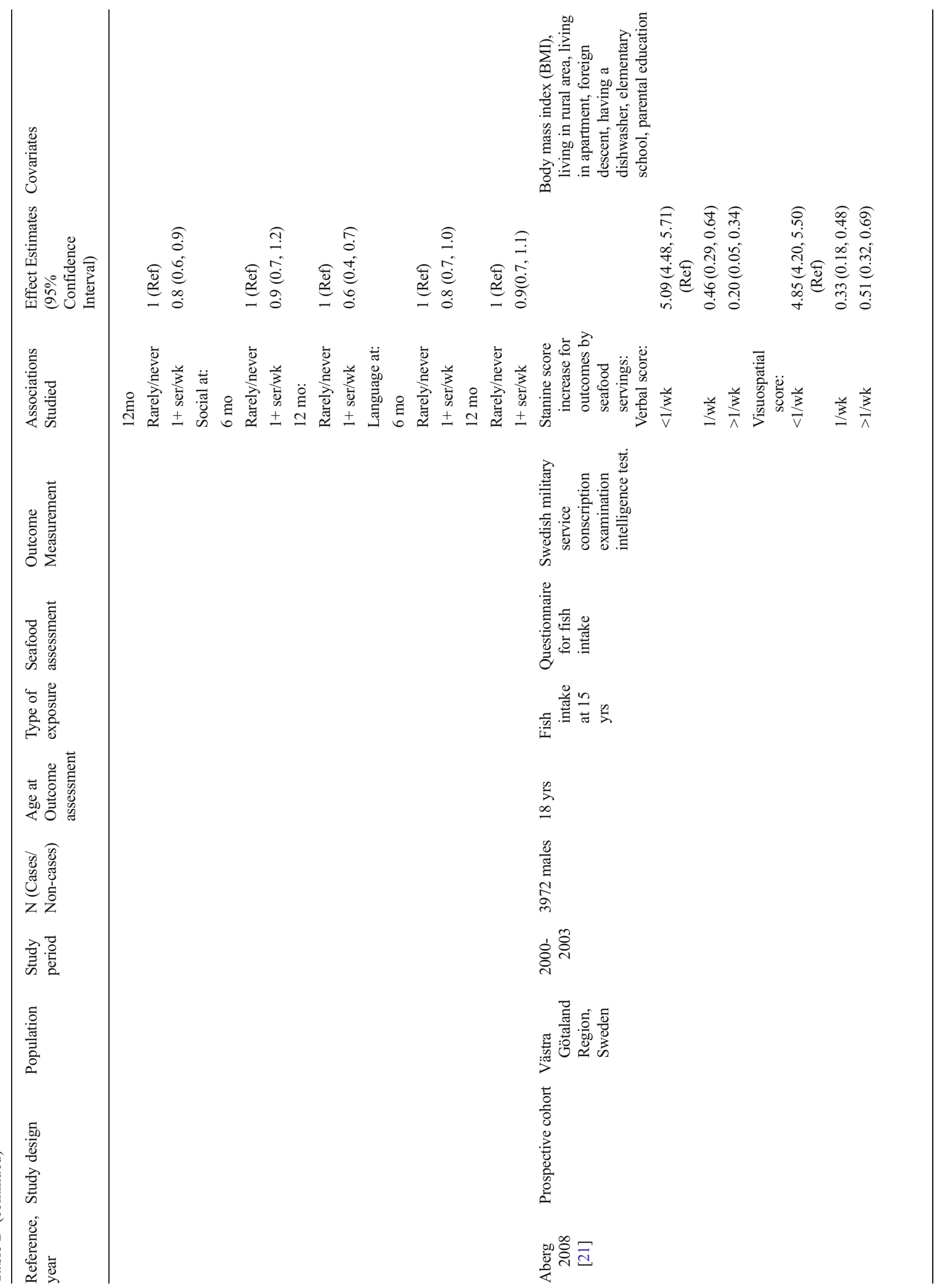



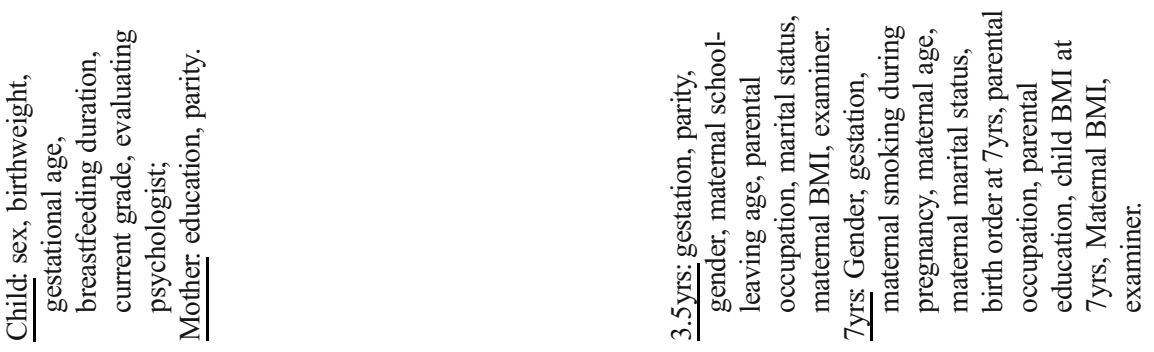

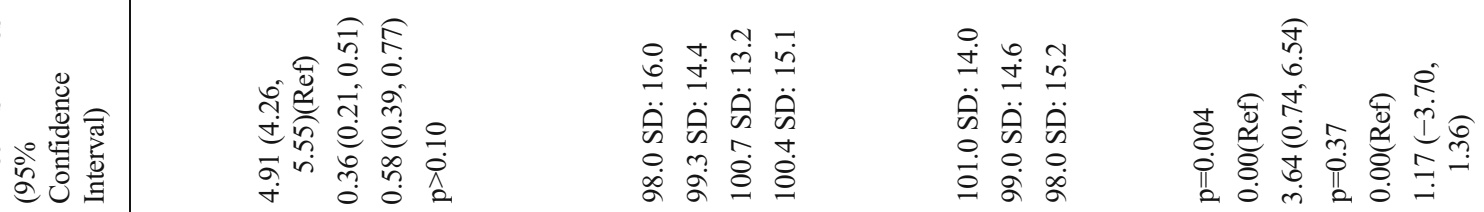

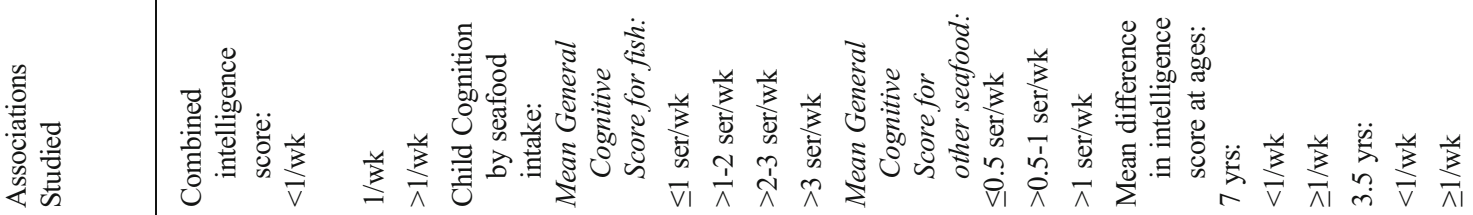

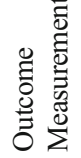

芯

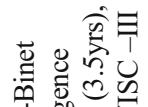

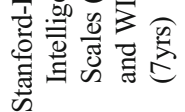

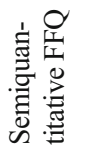

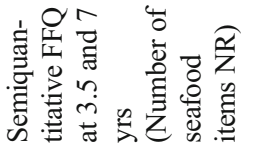

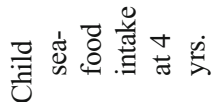

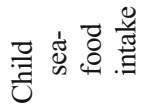

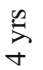

竎电

กิ

ळิ

至离离

ลั

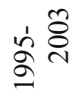

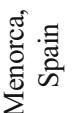

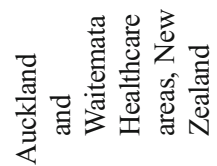

농

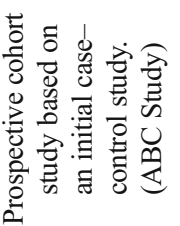

害

空

空运

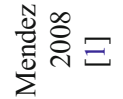

营 율를 


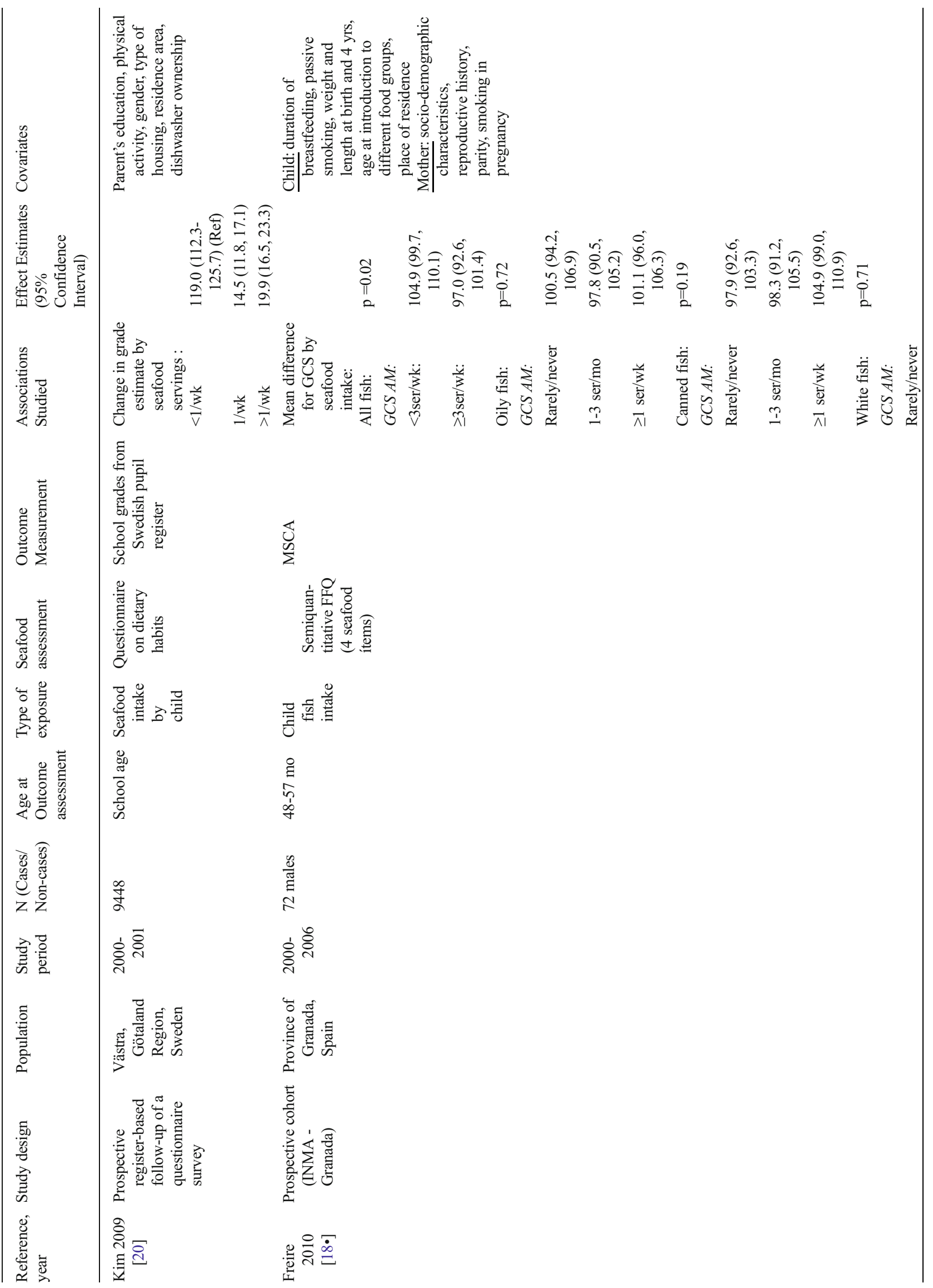




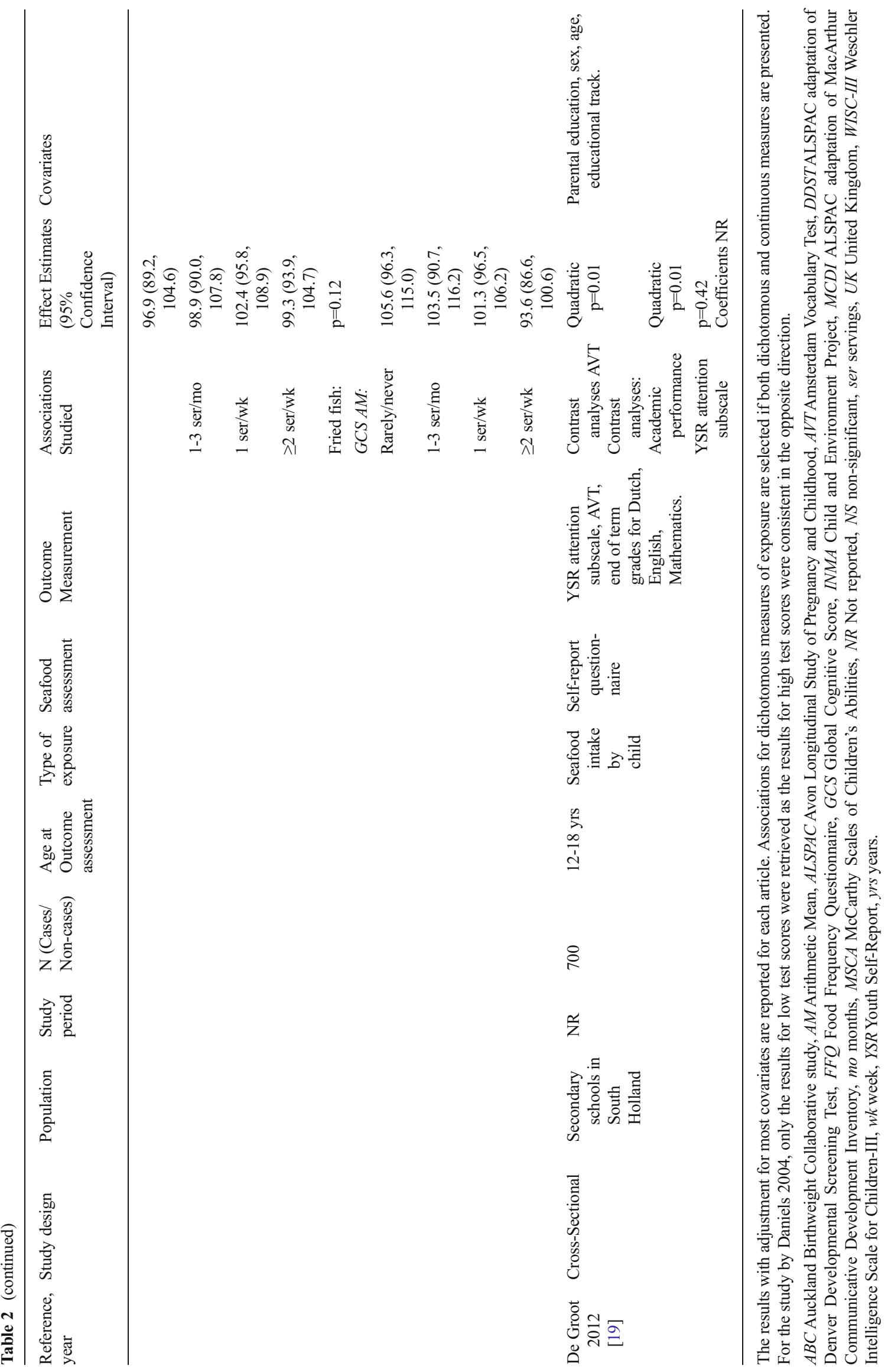


Table 3 Association between seafood intake and neurodevelopmental endpoints before and after adjustment for mercury and/or persistent organic pollutants

\begin{tabular}{|c|c|c|c|c|c|}
\hline Study & $\begin{array}{l}\text { Time of } \\
\text { Exposure }\end{array}$ & Association studied & $\begin{array}{l}\text { Effect size before } \\
\text { adjustment }\end{array}$ & $\begin{array}{l}\text { Effect size after } \\
\text { adjustment for } \\
\text { mercury or POPs }\end{array}$ & Model includes \\
\hline \multirow[t]{39}{*}{$\begin{array}{l}\text { Daniels, } 2004 \\
\quad[15]\end{array}$} & Prenatal & $\frac{\text { OR by seafood intake: }}{\text { MCDI Social Activity: }}$ & & $\begin{array}{l}\text { NR. (association } \\
\quad \text { remained similar) }\end{array}$ & Cord blood mercury concentrations \\
\hline & & Rarely/never & 1.00 (Ref) & & \\
\hline & & $1 / 2 \mathrm{wks}$ & $0.8(0.6,1.1)$ & & \\
\hline & & $1-3 / \mathrm{wk}$ & $0.6(0.5,0.8)$ & & \\
\hline & & $4+/ \mathrm{wk}$ & $0.7(0.5,0.9)$ & & \\
\hline & & $\begin{array}{l}\text { MCDI Vocabulary } \\
\text { comprehension: }\end{array}$ & & & \\
\hline & & Rarely/never & 1.00 (Ref) & & \\
\hline & & $1 / 2 \mathrm{wks}$ & $0.8(0.6,1.1)$ & & \\
\hline & & $1-3 / w k$ & $0.8(0.6,1.0)$ & & \\
\hline & & $4+/ \mathrm{wk}$ & $0.9(0.7,1.2)$ & & \\
\hline & & DDST Language: & & & \\
\hline & & Rarely/never & 1.00 (Ref) & & \\
\hline & & $1 / 2 \mathrm{wks}$ & $0.8(0.6,1.2)$ & & \\
\hline & & 1-3ser/wk: & $0.7(0.5-0.9)$ & & \\
\hline & & 4+ser/wk: & $0.7(0.5-0.9)$ & & \\
\hline & & DDST Social: & & & \\
\hline & & Rarely/never & 1.00 (Ref) & & \\
\hline & & $1 / 2$ wks & $1.2(0.8,1.8)$ & & \\
\hline & & $1-3 / \mathrm{wk}$ & $1.0(0.7-1.4)$ & & \\
\hline & & $4+/$ wk & $1.1(0.7-1.5)$ & & \\
\hline & & $\begin{array}{l}\frac{\text { Mean Developmental }}{\text { Assessment Scores by }} \\
\text { Meafood servings: } \\
\text { MCDI }\end{array}$ & & & \\
\hline & & Vocabulary Comprehension: & & & \\
\hline & & Rarely/never & $68.2(66.3,70.5)$ & & \\
\hline & & $1 / 2 \mathrm{wks}$ & $70.9(69.0,72.9)$ & & \\
\hline & & $1-3 / \mathrm{wk}$ & $73.0(71.2,74.8)$ & & \\
\hline & & $4+/$ wk & $71.9(70.5-73.8)$ & & \\
\hline & & $\begin{array}{l}\beta \pm \text { SD }(\mathrm{p}) \text { per ounce increase } \\
\text { of fish per wk } \\
M C D I\end{array}$ & $0.11 \pm 0.05(0.03)$ & & \\
\hline & & Social Activity: & & & \\
\hline & & Rarely/never & $16.4(16.0,16.7)$ & & \\
\hline & & $1 / 2 w k s$ & $17.0(16.6,17.3)$ & & \\
\hline & & $1-3 / w k$ & $17.1(16.8,17.4)$ & & \\
\hline & & $4+/ w k$ & $17.2(16.9,17.5)$ & & \\
\hline & & $\begin{array}{l}\beta \pm S D(p) \text { per ounce increase } \\
\text { of fish per wk }\end{array}$ & $\begin{array}{l}0.03 \pm 0.009 \\
(0.002)\end{array}$ & & \\
\hline & & $\underline{\text { DDST Total: }}$ & & & \\
\hline & & Rarely/never & $37.2(36.9,37.6)$ & & \\
\hline & & $1 / 2 w k s$ & $37.7(37.3,38.0)$ & & \\
\hline & & $1-3 / w k$ & $37.9(37.6,38.2)$ & & \\
\hline & & $4+/ w k$ & $37.8(37.5,38.1)$ & & \\
\hline & & $\begin{array}{l}\beta \pm S D(p) \text { per ounce increase } \\
\text { of fish per wk }\end{array}$ & $0.02 \pm 0.01(0.03)$ & & \\
\hline
\end{tabular}


Table 3 (continued)

\begin{tabular}{|c|c|c|c|c|c|}
\hline Study & $\begin{array}{l}\text { Time of } \\
\text { Exposure }\end{array}$ & Association studied & $\begin{array}{l}\text { Effect size before } \\
\text { adjustment }\end{array}$ & $\begin{array}{l}\text { Effect size after } \\
\text { adjustment for } \\
\text { mercury or POPs }\end{array}$ & Model includes \\
\hline & & \multicolumn{4}{|l|}{$\underline{D D S T \text { Language: }}$} \\
\hline & & Rarely/never & \multicolumn{3}{|l|}{$7.1(6.9,7.3)$} \\
\hline & & $1 / 2 w k s$ & \multicolumn{3}{|l|}{$7.4(7.2,7.5)$} \\
\hline & & $1-3 / w k$ & \multicolumn{3}{|l|}{$7.4(7.3,7.5)$} \\
\hline & & $4+/ w k$ & \multicolumn{3}{|l|}{$7.4(7.3,7.6)$} \\
\hline & & $\begin{array}{l}\beta \pm S D(p) \text { per ounce increase } \\
\text { of fish per wk }\end{array}$ & \multicolumn{3}{|l|}{$\begin{array}{l}0.01 \pm 0.004 \\
(0.004)\end{array}$} \\
\hline & & \multicolumn{4}{|l|}{ DDST Social: } \\
\hline & & Rarely/never & \multicolumn{3}{|l|}{$8.1(7.9,8.2)$} \\
\hline & & $1 / 2 w k s$ & \multicolumn{3}{|l|}{$8.1(8.0,8.2)$} \\
\hline & & $1-3 / w k$ & \multicolumn{3}{|l|}{$8.2(8.1,8.3)$} \\
\hline & & $4+/ w k$ & \multicolumn{3}{|l|}{$8.2(8.0,8.3)$} \\
\hline & & $\begin{array}{l}\beta \pm S D(p) \text { per ounce increase } \\
\text { of fish per wk }\end{array}$ & \multicolumn{3}{|l|}{$\begin{array}{l}0.002 \pm 0.004 \\
\quad(0.5)\end{array}$} \\
\hline $\begin{array}{l}\text { Oken, } 2005 \\
\text { [22] }\end{array}$ & Prenatal & $\begin{array}{c}\text { Change in VRM Score per } \\
\text { weekly seafood serving }\end{array}$ & $2.8(0.2,5.4)^{*}$ & $4.0(1.3,6.7)$ & Maternal hair mercury concentrations \\
\hline \multirow[t]{20}{*}{$\begin{array}{l}\text { Oken, } 2008 \\
\quad[24]\end{array}$} & Prenatal & \multicolumn{3}{|l|}{$\begin{array}{l}\text { Change in outcome by seafood } \\
\text { servings per wk: } \\
\text { PPVT score: }\end{array}$} & Maternal erythrocyte mercury concentrations \\
\hline & & $>2 / w k$ & $1.2(-3.5,6.0)^{*}$ & $2.2(-2.6,7.0)$ & \\
\hline & & $\leq 2 / \mathrm{wk}$ & $-2.1(-5.7,1.4)^{*}$ & $-1.8(-5.4,1.8)$ & \\
\hline & & Never & 0 (Ref) & 0 (Ref) & \\
\hline & & \multicolumn{4}{|l|}{ WRAVMA drawing score: } \\
\hline & & $>2 / \mathrm{wk}$ & $6.0(1.8,10.2)^{*}$ & $6.4(2.1,10.7)$ & \\
\hline & & $\leq 2 / \mathrm{wk}$ & $1.2(-2.0,4.4)^{*}$ & $1.3(-1.8,4.5)$ & \\
\hline & & Never & 0 (Ref) & 0 (Ref) & \\
\hline & & \multicolumn{4}{|l|}{ WRAVMA pegboard Score: } \\
\hline & & $>2 / \mathrm{wk}$ & $2.9(-1.4,7.1)^{*}$ & $3.5(-0.8,7.8)$ & \\
\hline & & $\leq 2 / \mathrm{wk}$ & $-0.7(-3.9,2.4)^{*}$ & $-0.5(3.7,2.7)$ & \\
\hline & & Never & 0 (Ref) & 0 (Ref) & \\
\hline & & \multicolumn{4}{|l|}{ WRAVMA matching score: } \\
\hline & & $>2 / w k$ & $2.8(-3.1,8.6)^{*}$ & $4.1(-1.8,10.0)$ & \\
\hline & & $\leq 2 / \mathrm{wk}$ & $1.8(-2.6,6.3)^{*}$ & $2.3(-2.1,6.7)$ & \\
\hline & & Never & 0 (Ref) & 0 (Ref) & \\
\hline & & \multicolumn{4}{|l|}{ WRAVMA total score: } \\
\hline & & $>2 / \mathrm{wk}$ & $5.3(0.9,9.6)^{*}$ & $6.4(2.0,10.8)$ & \\
\hline & & $\leq 2 / \mathrm{wk}$ & $1.1(-2.2,4.4)^{*}$ & $1.5(-1.8,4.7)$ & \\
\hline & & Never & 0 (Ref) & 0 (Ref) & \\
\hline \multirow[t]{9}{*}{$\begin{array}{l}\text { Mendez, } 2008 \\
\quad[1]\end{array}$} & $\begin{array}{l}\text { Pre and } \\
\text { Post- } \\
\text { natal }\end{array}$ & $\begin{array}{l}\text { Change in MSCA scores by } \\
\text { seafood servings } \\
\text { Breast-fed for }<6 m o \\
\end{array}$ & & $\begin{array}{l}\text { NR (association } \\
\quad \text { remained similar) }\end{array}$ & $\begin{array}{l}\text { Cord blood DDT, DDE, and PCB } \\
\text { concentrations were examined but } \\
\text { excluded from the final model }\end{array}$ \\
\hline & & General Cognitive: & & & \\
\hline & & $\leq 1 \mathrm{fish} / \mathrm{wk}$ & \multicolumn{3}{|l|}{0 (Ref) } \\
\hline & & $1-2 \mathrm{fish} / \mathrm{wk}$ & \multicolumn{3}{|l|}{$2.7(-1.2,6.5)$} \\
\hline & & 2-3 fish/wk & \multicolumn{3}{|l|}{$11.0(5.0,17.1)$} \\
\hline & & $>3$ fish/wk & \multicolumn{3}{|l|}{$-1.2(-9.8,7.3)$} \\
\hline & & \multicolumn{4}{|l|}{ Perceptual-Performance: } \\
\hline & & $\leq 1 \mathrm{fish} / \mathrm{wk}$ & \multicolumn{3}{|l|}{0 (Ref) } \\
\hline & & $1-2 \mathrm{fish} / \mathrm{wk}$ & $2.3(-1.5,6.1)$ & & \\
\hline
\end{tabular}


Table 3 (continued)

\begin{tabular}{|c|c|c|c|c|c|}
\hline Study & $\begin{array}{l}\text { Time of } \\
\text { Exposure }\end{array}$ & Association studied & $\begin{array}{l}\text { Effect size before } \\
\text { adjustment }\end{array}$ & $\begin{array}{l}\text { Effect size after } \\
\text { adjustment for } \\
\text { mercury or POPs }\end{array}$ & Model includes \\
\hline & & 2-3 fish/wk & $10.0(4.1,16.0)$ & & \\
\hline & & $>3$ fish/wk & $1.5(-7.0,9.9)$ & & \\
\hline & & Memory: & & & \\
\hline & & $\leq 1 \mathrm{fish} / \mathrm{wk}$ & $\mathrm{O}$ (Ref) & & \\
\hline & & $1-2$ fish/wk & $2.0(-2.1,6.1)$ & & \\
\hline & & 2-3 fish/wk & $10.5(4.1,16.9)$ & & \\
\hline & & $>3$ fish/wk & $-3.3(-12.4,5.8)$ & & \\
\hline & & Verbal: & & & \\
\hline & & $\leq 1 \mathrm{fish} / \mathrm{wk}$ & 0 (Ref) & & \\
\hline & & $1-2$ fish/wk & $2.2(-1.8,6.3)$ & & \\
\hline & & 2-3 fish/wk & $9.9(3.5,16.2)$ & & \\
\hline & & $>3 \mathrm{fish} / \mathrm{wk}$ & $-1.8(-10.8,7,2)$ & & \\
\hline & & Numeric: & & & \\
\hline & & $\leq 1 \mathrm{fish} / \mathrm{wk}$ & 0 (Ref) & & \\
\hline & & $1-2$ fish/wk & $2.1(-1.8,6.0)$ & & \\
\hline & & 2-3 fish/wk & $6.8(0.7,12.8)$ & & \\
\hline & & $>3$ fish/wk & $-2.3(-10.9,6.3)$ & & \\
\hline & & Motor Skills: & & & \\
\hline & & $\leq 1$ fish/wk & 0 (Ref) & & \\
\hline & & $1-2$ fish/wk & $2.1(-1.8,6.0)$ & & \\
\hline & & 2-3 fish/wk & $6.7(0.7,12.8)$ & & \\
\hline & & $>3$ fish/wk & $-2.3(-10.9,6.3)$ & & \\
\hline & & $\underline{\text { Breast-fed for } \geq 6 m o}$ & & & \\
\hline & & General Cognitive: & & & \\
\hline & & $\leq 1$ fish/wk & 0 (Ref) & & \\
\hline & & $1-2$ fish/wk & $-0.7(-7.0,5.7)$ & & \\
\hline & & 2-3 fish/wk & $-0.7(-8.3,6.9)$ & & \\
\hline & & $>3 \mathrm{fish} / \mathrm{wk}$ & $-5.3(-17.9,7.3)$ & & \\
\hline & & Perceptual-Performance: & & & \\
\hline & & $\leq 1 \mathrm{fish} / \mathrm{wk}$ & 0 (Ref) & & \\
\hline & & $1-2$ fish/wk & $-0.2(-6.1,6.6)$ & & \\
\hline & & 2-3 fish/wk & $0.8(-6.8,8.5)$ & & \\
\hline & & $>3$ fish/wk & $\begin{array}{c}-0.2(-12.4 \\
12.9)\end{array}$ & & \\
\hline & & Memory: & & & \\
\hline & & $\leq 1 \mathrm{fish} / \mathrm{wk}$ & 0 (Ref) & & \\
\hline & & $1-2$ fish/wk & $-1.8(-8.2,4.5)$ & & \\
\hline & & 2-3 fish/wk & $-4.6(-12.3,3.3)$ & & \\
\hline & & $>3$ fish/wk & $\begin{array}{l}-12.7(-25.5 \\
0.0)\end{array}$ & & \\
\hline & & Verbal: & & & \\
\hline & & $\leq 1 \mathrm{fish} / \mathrm{wk}$ & 0 (Ref) & & \\
\hline & & $1-2$ fish/wk & $-1.1(-7.7,5.4)$ & & \\
\hline & & 2-3 fish/wk & $-0.5(-8.3,7.4)$ & & \\
\hline & & $>3$ fish/wk & $-8.2(-21.3,4.9)$ & & \\
\hline & & Numeric: & & & \\
\hline & & $\leq 1 \mathrm{fish} / \mathrm{wk}$ & 0 (Ref) & & \\
\hline
\end{tabular}


Table 3 (continued)

\begin{tabular}{|c|c|c|c|c|c|}
\hline Study & $\begin{array}{l}\text { Time of } \\
\text { Exposure }\end{array}$ & Association studied & $\begin{array}{l}\text { Effect size before } \\
\text { adjustment }\end{array}$ & $\begin{array}{l}\text { Effect size after } \\
\text { adjustment for } \\
\text { mercury or POPs }\end{array}$ & Model includes \\
\hline & & $1-2 \mathrm{fish} / \mathrm{wk}$ & $-0.5(-6.8,5.9)$ & & \\
\hline & & $2-3$ fish/wk & $-3.1(-10.7,4.6)$ & & \\
\hline & & $>3$ fish/wk & $-2.8(-15.5,9.9)$ & & \\
\hline & & Motor Skills: & & & \\
\hline & & $\leq 1 \mathrm{fish} / \mathrm{wk}$ & 0 (Ref) & & \\
\hline & & $1-2$ fish/wk & $-2.1(-8.5,4.3)$ & & \\
\hline & & $2-3$ fish/wk & $-0.8(-8.5,7.0)$ & & \\
\hline & & $>3 \mathrm{fish} / \mathrm{wk}$ & $\begin{array}{l}-2.1(-14.8 \\
10.7)\end{array}$ & & \\
\hline \multirow[t]{19}{*}{$\begin{array}{l}\text { Freire, } 2010 \\
\quad[18 \bullet]\end{array}$} & Postnatal & $\begin{array}{l}\text { Change in General Cognitive } \\
\text { Score (MSCA) by seafood } \\
\text { servings: }\end{array}$ & $-7.9(\mathrm{p}=0.02)$ & & Child hair mercury concentrations \\
\hline & & $\begin{array}{l}\text { Oily fish: } \\
G C S A M \text { : }\end{array}$ & $\mathrm{p}=0.72$ & $\beta=1.02(-6.39,8.42)$ & \\
\hline & & Rarely/never & $\begin{array}{c}100.5(94.2 \\
106.9)\end{array}$ & & \\
\hline & & $1-3 \mathrm{ser} / \mathrm{mo}$ & $97.8(90.5,105.2)$ & & \\
\hline & & $\geq 1 \mathrm{ser} / \mathrm{wk}$ & $\begin{array}{c}101.1(96.0 \\
106.3)\end{array}$ & & \\
\hline & & $\begin{array}{l}\text { Canned fish: } \\
\text { GCS AM: }\end{array}$ & $\mathrm{p}=0.19$ & $\beta=7.98(0.28,15.68)$ & \\
\hline & & Rarely/never & $97.9(92.6,103.3)$ & & \\
\hline & & $1-3 \mathrm{ser} / \mathrm{mo}$ & $98.3(91.2,105.5)$ & & \\
\hline & & $\geq 1 \mathrm{ser} / \mathrm{wk}$ & $\begin{array}{c}104.9(99.0 \\
110.9)\end{array}$ & & \\
\hline & & $\begin{array}{l}\text { White fish: } \\
\text { GCS AM: }\end{array}$ & $\mathrm{p}=0.71$ & $\begin{array}{c}\beta=-3.32(-11.09 \\
4.46)\end{array}$ & \\
\hline & & Rarely/never & $96.9(89.2,104.6)$ & & \\
\hline & & $1-3 \mathrm{ser} / \mathrm{mo}$ & $98.9(90.0,107.8)$ & & \\
\hline & & $1 \mathrm{ser} / \mathrm{wk}$ & $\begin{array}{c}102.4(95.8 \\
108.9)\end{array}$ & & \\
\hline & & $\geq 2 \mathrm{ser} / \mathrm{wk}$ & $99.3(93.9,104.7)$ & & \\
\hline & & $\begin{array}{l}\text { Fried fish: } \\
\text { GCS AM: }\end{array}$ & $\mathrm{p}=0.12$ & $\begin{array}{l}\beta=-4.70(-12.34 \\
2.94)\end{array}$ & \\
\hline & & Rarely/never & $\begin{array}{c}105.6(96.3 \\
115.0)\end{array}$ & & \\
\hline & & $1-3 \mathrm{ser} / \mathrm{mo}$ & $\begin{array}{c}103.5(90.7 \\
116.2)\end{array}$ & & \\
\hline & & $1 \mathrm{ser} / \mathrm{wk}$ & $\begin{array}{c}101.3(96.5 \\
106.2)\end{array}$ & & \\
\hline & & $\geq 2 \mathrm{ser} / \mathrm{wk}$ & $93.6(86.6,100.6)$ & & \\
\hline $\begin{array}{l}\text { Suzuki, } 2010 \\
\quad[26]\end{array}$ & Prenatal & $\begin{array}{l}\text { Positive change in NBAS } \\
\text { motor cluster by maternal } \\
\text { seafood intake }\end{array}$ & $\begin{array}{l}\mathrm{p}=0.10 \\
\quad(\text { coefficients } \\
\text { NR) }\end{array}$ & $\begin{array}{l}\mathrm{p}<0.05^{* *} \\
\text { (coefficients NR) }\end{array}$ & $\begin{array}{l}\text { Maternal hair mercury concentrations } \\
\text { Cord blood PCB concentrations }\end{array}$ \\
\hline \multirow[t]{5}{*}{$\begin{array}{l}\text { Sagiv, } 2012 \\
{[27 \cdot]}\end{array}$} & Prenatal & $\frac{R R \text { for CRS-Teachers' version }}{\text { Inattentive }}$ & & & $\begin{array}{l}\text { Maternal hair mercury } \\
\text { Adjustment by cord serum PCB level NR }\end{array}$ \\
\hline & & $\leq 2 \mathrm{ser} / \mathrm{wk}$ & 1 (Ref) & 1 (Ref) & \\
\hline & & $>2 \mathrm{ser} / \mathrm{wk}$ & $0.6(0.4,0.8)^{*}$ & $0.6(0.4,0.9)$ & \\
\hline & & Impulsive/hyperactive & & & \\
\hline & & $\leq 2 \mathrm{ser} / \mathrm{wk}$ & 1 (Ref) & 1 (Ref) & \\
\hline
\end{tabular}


Table 3 (continued)

\begin{tabular}{|c|c|c|c|c|c|}
\hline \multirow[t]{2}{*}{ Study } & \multirow[t]{2}{*}{$\begin{array}{l}\text { Time of } \\
\text { Exposure }\end{array}$} & Association studied & $\begin{array}{l}\text { Effect size before } \\
\text { adjustment }\end{array}$ & $\begin{array}{l}\text { Effect size after } \\
\text { adjustment for } \\
\text { mercury or POPs }\end{array}$ & \multirow[t]{2}{*}{ Model includes } \\
\hline & & $>2$ ser $/ w k$ & $0.4(0.2,0.6)^{*}$ & $0.4(0.2-0.6)$ & \\
\hline & & Total & & & \\
\hline & & $\leq 2 \mathrm{ser} / \mathrm{wk}$ & 1 (Ref) & $1(\mathrm{Ref})$ & \\
\hline & & $>2 \mathrm{ser} / \mathrm{wk}$ & $(0.4,0.7)^{*}$ & $0.6(0.4,0.9)$ & \\
\hline & & $\frac{\text { Change for WISC-III by }}{\text { seafood intake: }}$ & & & \\
\hline & & $\leq 2 \mathrm{ser} / \mathrm{wk}$ & 0 (Ref) & 0 (Ref) & \\
\hline & & $>2$ ser $/$ wk & $1.3(-1.2,3.8)^{*}$ & $2.0(-0.8,4.8)$ & \\
\hline & & Freedom from distractibility & & & \\
\hline & & $\leq 2 \mathrm{ser} / \mathrm{wk}$ & 0 (Ref) & 0 (Ref) & \\
\hline & & $>2 \mathrm{ser} / \mathrm{wk}$ & $0.3(-1.9,2.6)^{*}$ & $1.5(-1.1,4.0)$ & \\
\hline & & $\underline{C P T:}$ & & & \\
\hline & & $\overline{\beta \text { for Mean reaction time }}$ & & & \\
\hline & & $\leq 2 \mathrm{ser} / \mathrm{wk}$ & 0 (Ref) & 0 (Ref) & \\
\hline & & $>2 \mathrm{ser} / \mathrm{wk}$ & $7.7(-3.8,19.3)^{*}$ & $10.1(-3.9,24.1)$ & \\
\hline & & $\beta$ for reaction time variability & & & \\
\hline & & $\leq 2 \mathrm{ser} / \mathrm{wk}$ & 0 (Ref) & 0 (Ref) & \\
\hline & & $>2$ ser $/$ wk & $(-3.6,6.7)^{*}$ & $-0.5(-6.3,5.4)$ & \\
\hline & & RR for Errors of Omission & & & \\
\hline & & $\leq 2 \mathrm{ser} / \mathrm{wk}$ & 1 (Ref) & 1 (Ref) & \\
\hline & & $>2$ ser/wk & $(0.7,1.2)^{*}$ & $(0.7,1.2)$ & \\
\hline & & RR for Errors of Comission & & & \\
\hline & & $\leq 2 \mathrm{ser} / \mathrm{wk}$ & 1 (Ref) & 1 (Ref) & \\
\hline & & $>2 \mathrm{ser} / \mathrm{wk}$ & $1.1(1.0,1.3)$ & $1.1(0.9,1.3)$ & \\
\hline
\end{tabular}

*After adjustment for participant characteristics ** Adjustment for toxicants but not participant characteristics

$A M$ Arithmetic Mean, DDE Dichlorodiphenyldichloroethylene, DDST ALSPAC adaptation of Denver Developmental Screening Test, DDT Dichlorodiphenyltrichlorethane, MCDI ALSPAC adaptation of MacArthur Children's Development Inventory, MSCA McCarthy Scales of Children's Abilities, NBAS Neonatal Behavioral Assessment Scale, PCB Polychlorinated Biphenyl, POPs Persitent Organic Pollutants, VRM Visual Recognition Memory, ser servings, wk week, $N R$ not reported

(Table 3), and WRAVMA at 3 years of age [24]. No significant differences were found for vocabulary using the PPVT [24]. However, results for processing speed, freedom from distractibility [27•], and performance IQ [25] have not shown clear associations.

With regards to behavioral outcomes, prenatal intake of fish was found to be inversely related to inattention, hyperactivity/impulsivity, and total score when evaluated by Conner's Rating Scale-Teacher's Version [27•]. An inverse association was also noted for hyperactivity as measured by SDQ [25]. No significant differences were found for other behavioral problems, peer problems, or emotional difficulties [25].

Some studies have evaluated different types of seafood [1, $16,18 \cdot, 24]$, showing that the type of seafood consumed during pregnancy can also be relevant. In a Spanish cohort, while fish was found to have positive effects, other kinds of seafood were associated with reduced cognitive scores [1] (Table 1). There may also be differences between fish types, such as was found in a study from the UK, in which the appearance of an effect of fish intake on behavior depended on the type of fish that had been consumed [25] (Table 1). In this case, only oily fish was inversely correlated with hyperactivity in childhood.

Few studies have evaluated the association of the same exposure with developmental outcomes on individuals with different characteristics. For example, a Spanish cohort study including 392 4-year-old children reported that prenatal fish intake of two or three servings per week was positively associated with higher IQ in children who had been breastfed for less than 6 months [1]. No association was found for children who had been breastfed for longer. However, no 
association or maybe even an inverse association was found for fish intake of three servings per week with no differences by breastfeeding. Maternal seafood intake may influence the neurodevelopment of their offspring not only through seafood consumption directly, but also through the relative composition of different food groups included in the diet. For instance, a Greek study [28] found that seafood intake was not associated with cerebral palsy, but an association was observed with the meat:fish dietary ratio during pregnancy.

\section{Studies of Seafood Intake During Childhood}

Seafood intake during childhood has been related to achieving better development [15], higher intelligence [17], and increased cognition scores [18•] in several studies. Other studies, however, reported no association between seafood intake during childhood and developmental outcomes. In a Spanish cohort, despite a positive tendency, no significant differences were found on the MSCA score according to child intake of seafood [1]. It may also be of importance to consider the type of fish that is eaten or the way it is prepared. In the INMAGranada subcohort [18•] oily and canned fish were positively associated, while white or fried fish were negatively associated with cognition. The same study found that when children consumed three or more servings of fish per week, general cognitive scores decreased, implying that the quantity of fish intake may also have a specific role.

\section{Studies of Seafood Intake during Adolescence}

We found three articles that considered the relationship between seafood intake and neurodevelopment during adolescence [19-21] (Table 2). In these studies, dietary exposures were measured approximately 1-3 years before evaluation. Seafood consumption during adolescence was associated with higher intelligence scores, better visuospatial performance [21], and improved school grades [20]. However, in one of the studies [19], despite a positive association of moderate consumption, the benefits of seafood intake disappeared at higher levels of seafood intake.

\section{Dose-Response Relationship}

Despite concern surrounding the possible negative effects of fish intake due to contamination with toxicants, most of the identified studies $[1,6-13,14 \bullet \cdot, 15-17,18 \bullet, 19,21]$ found a positive association between seafood intake and neurodevelopment outcomes. For example, in the ALSPAC study in the UK [10], a clear dose-response relationship was observed, higher amounts of seafood intake during pregnancy being associated with better developmental scores both before and after adjustment for covariates.
Although moderate seafood intake seemed beneficial across a majority of studies, some studies found that higher seafood intake could be correlated to lessening or reversal of the association between seafood and neurodevelopmental outcomes [1, 18•, 19, 25]. This has been described by De Groot as an upside-down Ushape relationship [19]. These findings may indicate that the relationship between seafood intake and cognition is not linear or that other negative influences strengthened by increasing the amount of seafood could counter its positive effects. Moreover, it is likely that the effects do not depend solely on the amount of seafood per se but on the type of seafood ingested $[18 \bullet, 25]$, which also raises the question whether the dose-response relationship varies between seafood types.

\section{Summary of Findings by Age of Outcome Examination}

A summary of the association between seafood intake and neurodevelopmental outcomes by the age of examination is shown in Table 4.

\section{Discussion}

This review shows a relatively small number of studies evaluating the association of seafood intake pre- and postnatally and neurodevelopment during childhood and adolescence. Most of the studies available were population-based prospective cohorts conducted in developed countries. Most studies showed positive associations between pre- and postnatal fish

Table 4 Association between seafood intake and neurodevelopment endpoints by age of outcome assessment

\begin{tabular}{ll}
\hline Age & Reported outcomes \\
\hline Infancy and & Marginally improved motor development [26] \\
toddlerhood & Better communicative and social development [10, 15] \\
& Higher infant cognition score [22] \\
& Higher developmental scores [23] \\
Childhood & Possible inverse association with cerebral palsy [28] \\
& Better visual cortex maturation and visual-motor \\
& development [16, 24] \\
& Better verbal IQ [10] \\
& Higher IQ [1, 17, 18•, 25] \\
& Less inattention [27•] \\
& Less hyperactivity/impulsivity [25, 27•] \\
& Improved social behavior [10] \\
& Attenuation/disappearance of positive association in \\
& highest seafood intake group [1, 18, 25$]$ \\
Better school grades [19, 20] & Higher IQ [21] \\
Better visuospatial performance [21] \\
Disappearance of positive association in the group with \\
higher than recommended seafood intake [19]
\end{tabular}


intake and neurodevelopment. The exposure measures were generally based on semiquantitative food frequency questionnaires reported by the mothers. The children's age at examination ranged from newborns to teenagers, and the outcome assessments included neurological, behavioral, and cognitive functioning. Most studies adjusted their models for sociodemographic characteristics. A few studies additionally controlled for the potential neurotoxic effect of pollutants related to seafood, such as methylmercury and POPs. An inverted U-shape trend was reported in some of the published work.

Semiquantitative food frequency questionnaires are wellestablished tools to assess seafood intake. They are widely used in standardized forms and have cross-cultural applicability [1]. Most of the studies reviewed here used them to assess seafood intake; however, there was no homogeneity in relation to the categorization of the exposure. The tendency was to adapt the frequency intake categories to the sample levels without following any global agreement based on international recommendations (i.e., UK's Food Standards Agency). However, a few studies used cutoff points of two servings per week or 340 g per week (Tables 1 and 2). An important number of studies only measured all seafood intake without going further in their analyses to evaluate different types separately, such as oily fish, white fish, and shellfish. It is unclear if this was due to the limited sample size or lack of data on type of fish intake frequency. The use of self-reported data is a major limitation in this field of research due to an increased level of noise related to the person's subjectivity in remembering food habits and the potential influence of individual's socio-cultural background. Additionally, healthy nutritional habits that include more fish intake are also related to higher maternal IQ and education level, and lower smoking habits during pregnancy $[24,25]$. We cannot rule out some residual confounding explaining the association with neurodevelopment. The assessment of some biomarkers of fish intake, such as serum docosahexaenoic acid (DHA) levels, strengthened only a couple of the reported seafood intake findings [14••, 16].

Fish intake was inversely associated with a variety of outcomes, including improved neurological functions and lower disorder prevalence such as superior foveal stereoacuity and cerebral palsy; better performances in cognitive functions related to verbal, memory, and visual-performance abilities; and improved behavioral outcomes related to hyperactivity, social competence, and school grades (Tables 1, 2, and 4). Even though the exact mechanisms explaining these benefits are not clear, these findings may indicate that the essential nutrients such as omega- 3 fatty acids derived from fish globally enhance neurodevelopment in both early and later stages. For instance, DHA may play an important role through several biological pathways during neuron differentiation and migration, axonal and synaptic growth, and synaptic connections until the early 20s. Deficit of dietary DHA is also known to alter neuronal myelination and cause mental retardation, and improvement is observed when DHA is restored [4, 29]. Additionally, animal studies have linked insufficiency of omega-3 fatty acids to alterations in neurotransmitters [30]. This could be related to behavioral and emotional changes.

Currently there is no agreement on which is the best-suited test to assess neurodevelopmental outcomes. Each study chose different tests and scales to assess similar functions. Heterogeneity of study outcomes will need to be considered in future projects trying to pool the data from different cohorts. We would suggest a harmonization of the tests in future studies. In relation to general cognitive assessments, it is important to follow tools that are internationally validated, but chosen depending on the age of the child such as the Bayley Scales during early life, followed by the MSCA scales or Wechsler Preschool and Primary Scale of Intelligence (WPPSI), WISC, and then Wechsler Adult Intelligence Scale (WAIS). Regarding less extended computer-based tests, we suggest following common paradigms in the neuropsychology [31] and using some of these tests: the CPT, Attention Network Task (ANT) or Stroop, Raven Colored Progressive Matrices, Trail Making Test (TMT), and N-BACK, for example. It is important not to use tests that may be culturally biased; one way is avoiding the assessment of verbal skills and school grades. Behavioral rating scales are more indirect measurements [32]; however, from these scales we can get important information about complementary behavioral areas during childhood, such as personality traits and social competence. Probably, the most widely used behavioral scales in epidemiological studies are the Child Behavior Check List (CBCL), SDQ, and Conner's Rating Scale.

A few studies observed an inverted U-shape pattern in the association between seafood intake and neurodevelopment (Tables 1 and 2), while most studies tended to show positive associations across all seafood categories. Those inverted Ushape studies attributed their findings to the higher pollutant exposure in the heavy seafood eaters (i.e., more than two servings per week), particularly if the type of fish is large and oily. However, oily fish also contains the highest DHA concentrations, which may counterbalance the neurotoxic effects. The few studies that were able to separate the exposure by type of seafood described conflicting results in relation to oily seafood type; in some cases oily fish was beneficial and in others it was less positive (Tables 1 and 2). Some studies argued that the sample size of the highest seafood intake category was too small to provide any conclusive finding [1]

Methylmercury and POPs are well-established neurotoxicants in populations where local seafood intake is the major dietary component ${ }^{2,116}$. The role of potential confounding by persistent pollutants such methylmercury and 
POPs in populations with low-moderate seafood intake, however, is less clear. In our review, the coefficients tended to increase after statistically controlling for these pollutants. Nevertheless, the coefficient change seems relatively small in relation to the overall beneficial association from seafood intake. Similar to the inverted U-shape trend among heavy seafood eaters, the strengthening of the association after adjustment for seafood pollutants could point to some potential counter-balanced effect from seafood toxicants. The high correlation between seafood intake and seafood pollutants, however, can make it very difficult to distinguish between the beneficial and detrimental effects of seafood intake, especially at low-moderate levels.

The general findings from the reviewed studies point toward a beneficial effect of consuming a moderate amount of seafood during pregnancy and early life. Overall, the observational studies included in this review support that a moderate amount of fish intake is beneficial to neurodevelopment. The findings from these observational studies are supported by a clinical trial that found higher scores in writing and reading tests after an intervention with a fish flour bread spread over a 6 -month period [14••]. The majority of studies included in this review were carried out in developed regions, and exposure to toxicants may be more important in some developing areas. On the other hand, it would also be wise to consider that in today's globalized market, the seafood that is consumed in one part of the world could easily come from a very different location.

As a precautionary approach, it is preferable during these life periods to avoid heavy intakes of large oily fish rich in methylmercury and POPs such as swordfish and tuna. This is important because of the observation in several studies of the attenuation or reversal of beneficial effects in the groups with the highest seafood consumption. The evidence gathered by this review suggests that the dose-response relationship is not linear for improved neurodevelopmental outcomes. This may be because of the increasing amounts of neurotoxicants ingested as more seafood is eaten; it may be that more toxic effects occur in diets with heavy seafood content. As to the type of seafood that should be recommended, this is not clear yet.

Information to the public should be managed with care. We recommend acknowledging the risk of exposure that large amounts of seafood per week can represent (especially of species with high mercury content), while also noting the benefits of moderate intake. If both aspects are not presented, individuals could limit their intake of seafood excessively, which not only reduces the ingestion of toxicants but also that of essential nutrients found in fish that appear to improve neurodevelopment. In a US study, the offspring of mothers who had followed US guidelines advising diets with less than
$340 \mathrm{~g}$ per week of fish were more likely to have suboptimum neurodevelopmental outcomes than the offspring of mothers who had consumed more than the recommended amount [10]. In this direction, governments and non-governmental institutions should collaborate in taking steps towards achieving equitable access to un-contaminated fish as part of a balanced diet, prioritizing pregnant women and children within socially, economically or environmentally vulnerable groups.

Larger cohort studies need to further address uncertainties and focus on wider ranges of frequency intakes and types of seafood. Using standard cutoffs based on international recommendations to classify the exposure and based on agreement on the neuropsychological tools used for assessments will help to increase comparability across studies, facilitating future efforts to merge the data for gaining power. Additionally, the measurements of fish intake biomarkers, such as blood DHA concentration, may help to disentangle the potential biological pathways and improve the validity of semiquantitative food frequency questionnaires. The use of neuroimaging in studies could also be contemplated in order to improve the understanding of the biological mechanisms through which fish intake is beneficial. Moreover, interventional studies are strongly required to rule out possible residual confounding and strengthen causality hypotheses.

\section{Conclusion}

Several studies conducted in the last 20 years support a beneficial role of seafood intake during neurodevelopment, both pre- and postnatally. Seafood intake benefits could cover an important range of behaviors, including neurological and cognitive functions, hyperactivity, social competence and school performance. Important questions remain, especially regarding the type of seafood, the shape of the dose response, and potential harmful exposures at the high end of seafood exposure. Larger cohort studies with more harmonized outcome assessments and detailed seafood intake information are needed to discriminate whether the positive associations are global or related to fish type and intake frequency. Interventional studies are strongly recommended. Finally and most importantly, these findings support the idea that steps should be taken to reduce contamination of water environments to continue having seafood as part of a healthy diet.

\section{Acknowledgments JCI-2011-09771 - MINECO}

The BREATHE project (BRain dEvelopment and Air polluTion ultrafine particles in scHool children) was funded by the

European Commission Seventh Framework Programme (FP7) (grant no. 268479)

Consorci MAR Parc de Salut de Barcelona - Ministerio de Sanidad, España. 


\section{Compliance with Ethics Guidelines}

Conflict of Interest Claudia B. Avella-Garcia and Jordi Julvez declare that they have no conflict of interest.

Human and Animal Rights and Informed Consent This article does not contain any studies with human or animal subjects performed by any of the authors.

\section{References}

Papers of particular interest, published recently, have been highlighted as:

- Of importance

•- Of major importance

1. Mendez MA, Torrent M, Julvez J, Ribas-Fito N, Kogevinas M, Sunyer J. Maternal fish and other seafood intakes during pregnancy and child neurodevelopment at age 4 years. Public Health Nutr. 2009;12(10):1702-10.

2. Grandjean PLP. Developmental neurotoxicity of industrial chemicals. Lancet. 2006;16(368):2167.

3. Mozaffarian DRE. Fish intake, contaminants, and human health: Evaluating the risks and the benefits. JAMA. 2006;296(15):188599. JAMA. 2006;296(15):1885.

4. Rombaldi Bernardi J, de Souza Escobar R, Ferreira C, Pelufo Silveira P. Fetal and neonatal levels of omega-3: Effects on neurodevelopment, nutrition, and growth. ScientificWorldJournal. 2012;202473.

5. Oken E, Guthrie L, Bloomingdale A, et al. A pilot randomized controlled trial to promote healthful fish consumption during pregnancy: The food for thought study. Nutr J. 2013;12(33).

6. Schoeman K, Bend J, Hill J, Nash K, Koren G. Defining a lowest observable adverse effect hair concentrations of mercury for neurodevelopmental effects of prenatal methylmercury exposure through maternal fish consumption: A systematic review. Ther Drug Monit. 2009;31(6):670.

7. Grandjean P, Satoh H, Murata K, Eto K. Adverse effects of methylmercury: Environmental health research implications. Environ Health Perspect. 2010;118(8):1137.

8. Jurewicz J, Hanke W. Prenatal and childhood exposure to pesticides and neurobehavioral development: Review of epidemiological studies. Int J Occup Med Environ Health. 2008;21(2):121.

9. Polańska K, Jurewicz J, Hanke W. Review of current evidence on the impact of pesticides, polychlorinated biphenyls and selected metals on attention deficit / hyperactivity disorder in children. Int J Occup Med Environ Health. 2013;26(1):16.

10. Hibbeln JR, Davis JM, Steer C, et al. Maternal seafood consumption in pregnancy and neurodevelopmental outcomes in childhood (ALSPAC study): An observational cohort study. Lancet. 2007;369 (9561):578-85.

11. Davidson PW, Leste A, Benstrong E, et al. Fish consumption, mercury exposure, and their associations with scholastic achievement in the Seychelles Child Development Study. Neurotoxicology. 2010;31(5):439-47.

12. Davidson PW, Cory-Slechta DA, Thurston SW, et al. Fish consumption and prenatal methylmercury exposure: Cognitive and behavioral outcomes in the main cohort at 17 years from the Seychelles Child Development Study. Neurotoxicology. 2011;32 (6):711-7.
13. Kinney DK, Teixeira P, Hsu D, et al. Relation of schizophrenia prevalence to latitude, climate, fish consumption, infant mortality, and skin color: A role for prenatal vitamin d deficiency and infections? Schizophr Bull. 2009;35(3):582-95.

14.• Dalton A, Wolmarans P, Witthuhn RC, van Stuijvenberg ME, Swanevelder SA, Smuts CM. A randomised control trial in schoolchildren showed improvement in cognitive function after consuming a bread spread, containing fish flour from a marine source. Prostaglandins Leukot Essent Fatty Acids. 2009;80(2-3):143-9. This study was the only randomized controlled study retrieved during our review of the literature on this subject.

15. Daniels JL, Longnecker MP, Rowland AS, Golding J. ALSPAC Study Team. University of Bristol Institute of Child Health. Fish intake during pregnancy and early cognitive development of offspring. Epidemiology. 2004;15(4):394-402.

16. Williams C, Birch EE, Emmett PM, Northstone K. Avon Longitudinal Study of Pregnancy and Childhood Study Team. Stereoacuity at age $3.5 \mathrm{y}$ in children born full-term is associated with prenatal and postnatal dietary factors: A report from a population-based cohort study. Am J Clin Nutr. 2001;73(2):316 22.

17. Theodore RF, Thompson JMD, Waldie KE, et al. Dietary patterns and intelligence in early and middle childhood. Intelligence. 2009;37:506.

18. Freire C, Ramos R, Lopez-Espinosa MJ, et al. Hair mercury levels, fish consumption, and cognitive development in preschool children from Granada, Spain. Environ Res. 2010;110(1):96-104. This paper studies cognitive outcomes in children in relation to fish intake. Of special note, it discriminates the results by the different types of fish consumed.

19. de Groot RH, Ouwehand C, Jolles J. Eating the right amount of fish: Inverted U-shape association between fish consumption and cognitive performance and academic achievement in Dutch adolescents. Prostaglandins Leukot Essent Fatty Acids. 2012;86(3):113-7.

20. Kim JL, Winkvist A, Aberg MA, et al. Fish consumption and school grades in Swedish adolescents: A study of the large general population. Acta Paediatr. 2010;99(1):72-7.

21. Aberg MA, Aberg N, Brisman J, Sundberg R, Winkvist A, Toren K. Fish intake of Swedish male adolescents is a predictor of cognitive performance. Acta Paediatr. 2009;98(3):555-60.

22. Oken E, Wright RO, Kleinman KP, et al. Maternal fish consumption, hair mercury, and infant cognition in a US cohort. Environ Health Perspect. 2005;113(10):1376-80.

23. Oken E, Osterdal ML, Gillman MW, et al. Associations of maternal fish intake during pregnancy and breastfeeding duration with attainment of developmental milestones in early childhood: A study from the Danish National Birth Cohort. Am J Clin Nutr. 2008;88 (3):789-96.

24. Oken E, Radesky JS, Wright RO, et al. Maternal fish intake during pregnancy, blood mercury levels, and child cognition at age 3 years in a US cohort. Am J Epidemiol. 2008;167 (10):1171-81.

25. Gale CR, Robinson SM, Godfrey KM, Law CM, Schlotz W, O'Callaghan FJ. Oily fish intake during pregnancy-association with lower hyperactivity but not with higher full-scale IQ in offspring. J Child Psychol Psychiatry. 2008;49(10):1061-8.

26. Suzuki K, Nakai K, Sugawara $T$, et al. Neurobehavioral effects of prenatal exposure to methylmercury and PCBs, and seafood intake: Neonatal behavioral assessment scale results of Tohoku Study of Child Development. Environ Res. 2010;110(7):699-704.

27. Sagiv SK, Thurston SW, Bellinger DC, Amarasiriwardena C, Korrick SA. Prenatal exposure to mercury and fish consumption during pregnancy and attention-deficit/hyperactivity 
disorder-related behavior in children. Arch Pediatr Adolesc Med. 2012;166(12):1123-31. This article discusses the beneficial effect of prenatal fish intake on child behavior, particularly ADHD related, while also taking mercury exposure into account.

28. Petridou E, Koussouri M, Toupadaki N, et al. Diet during pregnancy and the risk of cerebral palsy. Br J Nutr. 1998;79(5):407-12.

29. Martinez M. Restoring the DHA levels in the brains of Zellweger patients. J Mol Neurosci. 2001;16:309.
30. Kodas E, Galineau L. Bodard Sea. Serotoninergic neurotransmission is affected by n-3 polyunsaturated acids in the rat. $J$ Neurochem. 2004;89:695.

31. Baron SI. Neuropsychological evaluation of the child. 1st ed. New York: Oxford University Press; 2004. p. 429.

32. Julvez J, Forns M, Ribas-Fito N, et al. Psychometric characteristics of the California Preschool Social Competence Scale in a Spanish population sample. Early Educ Dev. 2008;19(5):795. 